\title{
MUSE GAS FLOW AND WIND (MEGAFLOW). I. FIRST MUSE RESULTS ON BACKGROUND QUASARS*
}

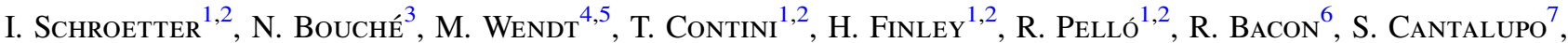 \\ R. A. Marino ${ }^{7}$, J. Richard ${ }^{6}$, S. J. Lilly ${ }^{7}$, J. Schaye ${ }^{8}$, K. Soto ${ }^{7}$, M. Steinmetz ${ }^{4}$, L. A. Straka ${ }^{8}$, And L. Wisotzki ${ }^{4}$ \\ ${ }^{1}$ IRAP, Institut de Recherche en Astrophysique et Planétologie, CNRS, 14, avenue Edouard Belin, F-31400 Toulouse, France \\ ${ }^{2}$ University Paul Sabatier of Toulouse/UPS-OMP/IRAP, F-31400 Toulouse, France \\ ${ }^{3}$ IRAP/CNRS, 9, avenue Colonel Roche, F-31400 Toulouse, France \\ ${ }^{4}$ AIP, Leibniz-Institut für Astrophysik Potsdam, An der Sternwarte 16, D-14482 Potsdam, Germany \\ ${ }^{5}$ Institut für Physik und Astronomie, Universität Potsdam, D-14476 Golm, Germany \\ ${ }^{6}$ Univ Lyon, Univ Lyon1, Ens de Lyon, CNRS, Centre de Recherche Astrophysique de Lyon UMR5574, F-69230, Saint-Genis-Laval, France \\ ${ }^{7}$ ETH Zurich, Institute of Astronomy, Wolfgang-Pauli-Str. 27, 8093 Zurich, Switzerland \\ ${ }^{8}$ Leiden Observatory, Leiden University, P.O. Box 9513, 2300 RA Leiden, The Netherlands \\ Received 2016 May 4; revised 2016 September 15; accepted 2016 September 30; published 2016 December 6
}

\section{ABSTRACT}

The physical properties of galactic winds are one of the keys to understand galaxy formation and evolution. These properties can be constrained thanks to background quasar lines of sight (LOS) passing near star-forming galaxies (SFGs). We present the first results of the MusE GAs FLOw and Wind survey obtained from two quasar fields, which have eight $\mathrm{Mg}$ II absorbers of which three have rest equivalent width greater than $0.8 \AA$. With the new Multi Unit Spectroscopic Explorer (MUSE) spectrograph on the Very Large Telescope (VLT), we detect six (75\%) Mg II host galaxy candidates within a radius of $30^{\prime \prime}$ from the quasar LOS. Out of these six galaxy-quasar pairs, from geometrical argument, one is likely probing galactic outflows, where two are classified as "ambiguous," two are likely probing extended gaseous disks and one pair seems to be a merger. We focus on the wind-pair and constrain the outflow using a high-resolution quasar spectra from the Ultraviolet and Visual Echelle Spectrograph. Assuming the metal absorption to be due to ga; s flowing out of the detected galaxy through a cone along the minor axis, we find outflow velocities in the order of $\approx 150 \mathrm{~km} \mathrm{~s}^{-1}$ (i.e., smaller than the escape velocity) with a loading factor, $\eta=\dot{M}_{\text {out }} / \mathrm{SFR}$, of $\approx 0.7$. We see evidence for an open conical flow, with a low-density inner core. In the future, MUSE will provide us with about 80 multiple galaxy-quasar pairs in two dozen fields.

Key words: galaxies: evolution - galaxies: formation - intergalactic medium - quasars: individual (SDSS J213748 +001220 , SDSS J215200+062516)

\section{INTRODUCTION}

In spite of the successes of the $\Lambda \mathrm{CDM}$ cosmological model (i.e., Springel et al. 2005), a major discrepancy remains between the predicted number density of dark matter halos and the observed number density of galaxies in the low-mass regime $\left(L<L_{*}\right)$ (i.e., Guo et al. 2010; Papastergis et al. 2012; Moster et al. 2010, 2013; Behroozi et al. 2013). This behavior is usually explained by supernova (SN)-driven outflows (Dekel \& Silk 1986), which expel baryons from the galactic disk. Indeed, these galactic outflows are observed in almost every star-forming galaxy (SFG) (Veilleux et al. 2005, for a review) and are likely to enrich the intergalactic medium (e.g., Dekel \& Silk 1986; Aguirre et al. 2001; Oppenheimer \& Davé 2006).

The physical mechanisms for driving galactic winds are complex and the cold gas could be accelerated by thermal energy injection (Springel \& Hernquist 2003), by momentum injection from radiation pressure (e.g., Murray et al. 2005), by cosmic ray pressure (e.g., Booth et al. 2013; Salem \& Bryan 2014) or by a combination of these mechanisms (e.g., Hopkins 2015). The wide range of physical scales, which describe SN explosions from astronomical units to tens of kiloparsecs (kpc), are beyond the capabilities of cosmological simulations.

Hence, in most of these simulations, outflows are usually implemented with subgrid prescriptions (e.g., Oppenheimer et al. 2010; Schaye et al. 2010; Vogelsberger et al. 2014). A popular subgrid recipe is to let the loading factor $\eta$, i.e., the ratio between

* Based on observations made at the ESO telescopes under programs 094.A0211(B) and 293.A-5038(A). the outflow rate $\dot{M}_{\text {out }}$ and the star formation rate (SFR), be a function of galaxy (halo) mass or circular velocity $V_{c}$ (Oppenheimer et al. 2010) such as $\eta \propto V_{c}^{-1}$ for momentum-driven winds and $\eta \propto V_{c}^{-2}$ for energy-driven winds. An alternative way to implement the collective effect of $\mathrm{SN}$ explosions is the (stochastic) implementation of thermal feedback, where galactic winds develop without imposing any input outflow velocity or mass loading factor such as in the EAGLE simulations (e.g., Schaye et al. 2015), the FIRE simulations (Hopkins et al. 2014; Muratov et al. 2015), and the multi-phase scheme of Barai et al. (2015).

Given the high impact of SN feedback on galaxy formation and the wide range of mass loading factors used in numerical simulations (see the compilations in Torrey et al. 2014; Zahid et al. 2014; Schroetter et al. 2015), observational constraints are of paramount importance. Unfortunately, our knowledge of the loading factor or the mass outflow rate $\dot{M}_{\text {out }}$ is incomplete despite the many efforts made in the past decades (i.e., Lehnert \& Heckman 1996; Martin 1998, 1999; Heckman et al. 2000; Rupke et al. 2005; Rubin et al. 2010; Martin et al. 2012). Indeed, estimates of the ejected mass flux $\dot{M}_{\text {out }}$ using standard galaxy absorption lines (e.g., Heckman et al. 1990, 2000; Martin et al. 2002, 2012, 2013; Pettini et al. 2002; Martin 2005) are uncertain by orders of magnitude mainly due to the difficulty in constraining the location of the probed outflowing gas. ${ }^{10}$ Indeed, the gas responsible for the blueshifted absorption lines in galaxies could be $0.1,1$, or $10 \mathrm{kpc}$ away from the host. Some recent studies have

\footnotetext{
${ }^{10}$ Furthermore, outflow rates from these low-ionization metal lines also require uncertain ionization corrections (e.g., Chisholm et al. 2016).
} 
made serious attempts at determining the scaling of outflow rates with galaxy properties by setting the absorbing gas at a fixed distance (Chisholm et al. 2015; Heckman et al. 2015; Wood et al. 2015).

Background quasars can give us the minimum distance of the gas from the impact parameter $b$ and, thereby, potentially yield more accurate outflow rates (Bouché et al. 2012; Kacprzak et al. 2014; Muzahid et al. 2015; Schroetter et al. 2015). One difficulty is that it is rare for the lines of sight (LOS) to a background quasar to pass near a SFG. Hence, one needs to devise strategies to build large samples of galaxyquasar pairs. Another difficulty is that background quasars can probe not only the circumgalactic medium, but also the outer regions of gaseous disks and the gas near other, undetected galaxies.

In order to obtain large samples of galaxy-quasar pairs, one can select quasars around galaxies or galaxies around quasars with absorption systems. The former requires quasar followup observations, while the latter requires one to detect the associated galaxies. In the era of large quasar catalogs from the Sloan Digital Sky Survey (SDSS), we favor the absorptionselection technique combined with integral field unit (IFU) observations. Indeed, from $\mathrm{Mg}$ II absorption-selected quasar spectra, because we know the host galaxy redshift without knowing its position, IFUs can detect galaxies at previously unknown impact parameters. This kind of instrument also allows us to determine geometrical and kinematic properties of galaxies in the same observation. So far, IFUs such as SINFONI allowed us to probe galaxies within $20 \mathrm{kpc}$ from the quasar LOS (at redshift around 1). With the new Very Large Telescope (VLT)/Multi Unit Spectroscopic Explorer (MUSE) instrument (Bacon et al. 2006, 2009), one can now detect galaxies further away $(\sim 250 \mathrm{kpc}$ away at $z=1)$ thanks to its field of view of $1 \times 1$ arcmin (compared to $8^{\prime \prime} \times 8^{\prime \prime}$ for SINFONI). The large wavelength coverage of MUSE (4800-9300 A) allows us to target quasar fields with multiple Mg II $(\lambda \lambda 2796,2802)$ absorption lines having redshifts from $0.4-1.4$ for $[\mathrm{O}$ II $](\lambda \lambda 3727,3729)$ identification. We complement the VLT/MUSE IFU observations (which have a resolution $R \sim 2000$ or $150 \mathrm{~km} \mathrm{~s}^{-1}$ ) with VLT/Ultraviolet and Visual Echelle Spectrograph (UVES) followup high-resolution spectra of the quasars in order to study the LOS kinematics with the resolution $\left(<10 \mathrm{~km} \mathrm{~s}^{-1}\right)$ necessary for obtaining accurate constraints on outflow properties.

In this paper, we present the first results on galactic outflows from our MUSE survey. In Section 2, we present the survey, the MUSE+UVES data and the data reduction. Section 3 describes the sample results, while Section 4 presents our wind model as well as individual galaxy properties. Conclusions are then discussed in Section 5.

We use the $\Lambda$ CDM standard cosmological parameters: $H_{0}=70 \mathrm{~km} \mathrm{~s}^{-1}, \Omega_{\Lambda}=0.7$, and $\Omega_{M}=0.3$.

\section{THE MEGAFLOW SURVEY}

\subsection{Target Selection Strategy}

Current samples of galaxy-quasar pairs for strong $\mathrm{Mg}$ II absorbers, as in Bouché et al. (2012), Schroetter et al. (2015), Muzahid et al. (2015) and Bouché et al. (2016), are made of a dozen pairs. Here, we seek to increase the sample size by almost an order of magnitude in order to allow for statistical analysis of the relation between the absorption properties (and
Table 1

Summary of MUSE 094.A-0211(B) Observations

\begin{tabular}{lcccc}
\hline \hline $\begin{array}{l}\text { Field } \\
(1)\end{array}$ & $\begin{array}{c}z_{\text {qso }} \\
(2)\end{array}$ & $\begin{array}{c}\mathrm{PSF}\left({ }^{\prime \prime}\right) \\
(3)\end{array}$ & $\begin{array}{c}\mathrm{T}_{\text {exp }}(\mathrm{s}) \\
(4)\end{array}$ & $\begin{array}{c}\text { Date } \\
(5)\end{array}$ \\
\hline $\mathrm{J} 213748+0012$ & 1.668 & 0.8 & 3600 & 2014 Sep 23 \\
$\mathrm{J} 215200+0625$ & 2.409 & 0.7 & 7200 & 2014 Sep 24 \\
\hline
\end{tabular}

Note. (1) Quasar name; (2) Quasar emission redshift; (3) FWHM of the seeing point-spread function (PSF) (at $\approx 7000 \AA$ ); (4) Exposure time; (5) Date of observations.

ultimately wind properties such as outflow rates and loading factors) and the galaxy properties. Thanks to the multiplexing capabilities of MUSE, having a sample 80-100 pairs is now within reach using 20-25 quasar fields.

As in our previous surveys, we first select background quasar spectra with Mg II $\lambda 2796$ absorption lines. For our MusE GAs FLOw and Wind (MEGAFLOW) survey, our strategy consists in selecting multiple Mg II absorbers (three, four, or five) in quasar spectra from the Zhu and Ménard catalog $^{11}$ (Zhu \& Ménard 2013) based on the SDSS survey (Ross et al. 2012; Alam et al. 2015). These Mg II absorptions should have redshifts between 0.4-1.4 such that the [O II] $\lambda \lambda 3727,3729$ galaxy emission lines fall into the MUSE wavelength range (4800-9300 ̊).

To restrict the impact parameter range, we constrain the rest equivalent width (REW) of these absorptions $W_{r}^{\lambda 2796}$ to $W_{r}^{\lambda 2796}>0.5 \AA$, because of the well known anticorrelation between the impact parameter and $W_{r}^{\lambda 2796}$ (Lanzetta \& Bowen 1990; Steidel 1995; Chen et al. 2010; Bordoloi et al. 2011; Kacprzak et al. 2011b; Nielsen et al. 2013; Werk et al. 2013). Also, the largest $W_{r}^{\lambda 2796}$ tends to be associated with outflows (e.g., Kacprzak et al. 2011b; Lan et al. 2014). We define a strong absorber to be an absorber with $W_{r}^{\lambda 2796}>0.3-0.5 \AA$ as in Nestor et al. (2005). This limit of $0.5 \AA$ corresponds to $b \lesssim 100 \mathrm{kpc}$. We also need to pay attention to where the galaxy emission lines will appear in the spectrum and try to avoid bright sky emission lines as much as possible.

The MEGAFLOW survey will consist of 20-25 quasar fields and the MUSE observations started in 2014 September. In 2014 October, we obtained UVES observations on the first two fields (Table 1). ${ }^{12}$ In this paper, we present the first results on these two fields toward SDSS J213748+0012 and SDSS $\mathrm{J} 215200+0625$, which have four Mg II absorption systems each.

\subsection{Observations and Data Reduction}

\subsubsection{MUSE Observations}

MUSE data were taken in 2014 September in visitor mode during the first Guaranteed Time Observations (GTO) run (program ID 0.94A-0211). We first point the telescope toward a quasar and then we offset the first exposure by $\approx 4^{\prime \prime}-5^{\prime \prime}$ in R.A. and decl. This first offset is made to avoid the quasar flux falling in the same pixels as the first pointing. Each observation was composed of four exposures of $900 \mathrm{~s}$ with a rotation of $90^{\circ}$ between every exposure as well as small dithering $\left(<1^{\prime \prime}\right)$. This

\footnotetext{
11 This catalog can be found at http://www.pha.jhu.edu/ gz323/Site/ Download_Absorber_Catalog.html.

12 As Director Discretionary Time (DDT) program 293.A-5038(A).
} 
Table 2

Magnitude Differences between MUSE and SDSS for J213748+0012 and J215200+0625 Fields

\begin{tabular}{|c|c|c|c|c|c|c|c|}
\hline $\begin{array}{l}\text { Field } \\
\text { (1) }\end{array}$ & $\begin{array}{l}\text { Object } \\
(2)\end{array}$ & $\begin{array}{l}\text { Instrument } \\
\text { (3) }\end{array}$ & $\begin{array}{l}\text { R.A. } \\
\text { (4) }\end{array}$ & $\begin{array}{l}\text { Decl. } \\
(5)\end{array}$ & $\begin{array}{c}\operatorname{mag}_{r} \\
(6)\end{array}$ & $\begin{array}{c}\operatorname{mag}_{i} \\
(7)\end{array}$ & $\begin{array}{c}\text { Difference } \\
\text { (8) }\end{array}$ \\
\hline \multirow[t]{3}{*}{$\mathrm{J} 213748+0012$} & \multirow[t]{2}{*}{ QSO } & MUSE & $21: 37: 48.41$ & $+00: 12: 20.49$ & 18.33 & 18.19 & \multirow[t]{2}{*}{-0.13} \\
\hline & & SDSS & $21: 37: 48.44$ & $+00: 12: 20.00$ & 18.20 & 18.05 & \\
\hline & Star & SDSS & $21: 37: 47.65$ & $+00: 12: 20.89$ & 19.61 & 19.46 & -0.09 \\
\hline \multirow[t]{2}{*}{$\mathrm{J} 215200+0625$} & QSO & MUSE & 21:52:00.05 & $+06: 25: 17.26$ & 19.42 & 19.44 & -0.07 \\
\hline & Star & SDSS & 21:51:59.83 & $+06: 25: 04.72$ & 16.53 & 16.29 & -0.17 \\
\hline
\end{tabular}

Note. (1) Field; (2) Object type; (3) Instrument (MUSE or SDSS); (4) Right Ascension (R.A.); (5) Declination (Decl.); (6) Magnitude in $r$ filter (central wavelength $\lambda_{r}=6165 \AA$ ); (7) Magnitude in $i$ filter (central wavelength $\lambda_{i}=7481 \AA$ ); (8) Average difference SDSS-MUSE (mag).

Table 3

Summary of UVES 293.A-5038(A) Observations

\begin{tabular}{lccc}
\hline \hline Target & Setting $\lambda_{c}(\mathrm{~nm})$ & $\mathrm{T}_{\exp }(\mathrm{s})$ & Date \\
\hline $\mathrm{J} 213748+0012$ & $390+580$ & 5970 & 2014 Oct 19 \\
$\mathrm{J} 215200+0625$ & $390+580$ & 9015 & 2014 Oct 21, 24, 2014 \\
& & & Nov 18 \\
\hline
\end{tabular}

Table 4

Summary of MUSE Galaxy Detection

\begin{tabular}{lcccc}
\hline \hline $\begin{array}{l}\text { Field name } \\
(1)\end{array}$ & $\begin{array}{c}z_{\text {absorber }} \\
(2)\end{array}$ & $\begin{array}{c}W_{r}^{\lambda 2796} \\
(3)\end{array}$ & $\begin{array}{c}N_{\text {det }} \\
(4)\end{array}$ & $\begin{array}{c}b \\
(5)\end{array}$ \\
\hline $\mathrm{J} 213748+0012$ & 0.8063 & $0.724 \pm 0.09$ & 1 & 88 \\
& 1.0437 & $0.767 \pm 0.08$ & $0^{\mathrm{a}}$ & $\ldots$ \\
& 1.1890 & $0.308 \pm 0.06$ & 1 & 63 \\
$\mathrm{~J} 215200+0625$ & 1.2144 & $1.144 \pm 0.06$ & 3 & $87,212,246$ \\
& 1.0534 & $0.522 \pm 0.14$ & 2 & 45,189 \\
& 1.1761 & $0.526 \pm 0.15$ & 0 & $\cdots$ \\
& 1.3190 & $1.347 \pm 0.12$ & 1 & 34 \\
& 1.4309 & $1.152 \pm 0.11$ & 4 & $62,78,184,211$ \\
\hline
\end{tabular}

Notes. (1) Quasar field name; (2) Mg II absorption lines' redshift; (3) Mg II

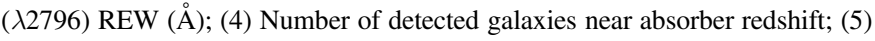
Impact parameter(s) of the detected galaxy(ies) (kpc).

${ }^{a}$ Affected by $\mathrm{OH}$ emission line at $7618 \AA$.

observation strategy is used in order to minimize systematics. From each MUSE observation, we obtain a combined cube of $317 \times 316$ spatial pixels (spaxels). Each spaxel has $\sim 3680$ spectral pixels ranging from 4750-9350 $\mathrm{A}$. With a spectral sampling of $1.25 \AA$ pixel $^{-1}$, the average spectral resolution of the data is $\sim 2.4 \AA$ FWHM. The spatial resolution for the two quasar fields is $\sim 0$ ". 8 FWHM with spatial sampling of $0 . \prime 2$ pixel $^{-1}$ at $7000 \AA$. The seeing constraint $(<0$ ". 9$)$ is necessary if we want to derive galaxy parameters and detect them. Indeed, galaxies at redshift $\sim 1$ can be small $(<1$ !'2) and we need the seeing to be smaller than the galaxy to better derive its parameters.

\subsubsection{MUSE Data Reduction}

The data are reduced using version 1.0 of the MUSE data reduction software (DRS) pipeline. ${ }^{13} \mathrm{We}$ process bias, flat-field calibrations and arc lamp exposures taken during the night of the observations. Following calibration processing, raw science

\footnotetext{
${ }^{13}$ A short description of the pipeline is given in Weilbacher et al. (2014).
}

frames are bias subtracted and flat-fielded using master bias and master flat fields respectively. The flat-fielding is renormalized in each slice to account for slight changes due to temperature variations using a single flat-field exposure taken hourly before the science observation or when the instrument temperature changes by more than $0.5^{\circ} \mathrm{C}$. An additional flat-field correction was performed using the twilight sky exposures taken at the beginning of each night to correct for slight optical path differences between sky and calibration unit. Geometrical calibration and astrometric solution are then applied. The wavelength solution is obtained from the arc lamps and calibrated in air. Wavelengths are also corrected for the heliocentric velocity. The flux calibration is obtained from a spectrophotometric star observed for each night.

On each individual exposure, we use the default configuration of the DRS recipe and with the sky removal method turned off. This produces, for the four individual exposures, a large table called the "pixel-table." For each individual exposure, star positions were registered in order to have accurate relative astrometry as shifts can occur between exposures due to the derotator wobble $(<0$ !! 3$)$. The "pixel-tables" were then combined into a single data cube using the previously calculated offsets. The sky subtraction was performed on this combined data cube with Zurich Atmosphere Purge (ZAP), an algorithm developed by Soto et al. (2016a, 2016b). ZAP operates by first subtracting a baseline sky level, found by calculating the median per spectral plane and then uses principal component analysis and determines the minimal number of eigenspectra, which can reconstruct the residual emission features in the data cube. Absolute astrometry is obtained by matching the positions of point sources in the data cube against the SDSS astrometry.

Finally, we cross-checked the flux calibration of these point sources against the SDSS magnitudes in the $r$ and $i$ filter bands (the central wavelengths are $\lambda_{r}=6165 \AA$ and $\lambda_{i}=7481 \AA$ for $r$ and $i$ filters, respectively), whose bandpasses are within the MUSE wavelength coverage. Using the $r$ and $i$ images obtained from the MUSE data cube convolved with the SDSS filters, we fitted a Moffat profile on each of the stars to calculate their total flux in each filter and then compare them with the SDSS ones. SDSS filters are designed to be in $\mathrm{AB}$ magnitudes, but there are still corrections needed for some filters. Given that for the $r$ and $i$ filters, the AB to SDSS magnitudes correction is negligible, we can correct fluxes into $\mathrm{AB}$ magnitudes directly using the 
Table 5

Surface Brightness and Flux Limits

\begin{tabular}{|c|c|c|c|c|c|c|}
\hline $\begin{array}{l}\text { Quasar field } \\
\text { (1) }\end{array}$ & $\begin{array}{c}z_{\text {absorber }} \\
\text { (2) }\end{array}$ & $\begin{array}{l}\mathrm{LSF} \\
(3)\end{array}$ & $\begin{array}{l}\text { Noise } \\
(4)\end{array}$ & $\begin{array}{c}\text { PSF } \\
(5)\end{array}$ & $\begin{array}{c}\text { Surface Brightness Limit } \\
(6)\end{array}$ & $\begin{array}{l}\text { [O II] Flux Limit } \\
\text { (7) }\end{array}$ \\
\hline $\mathrm{J} 213748+0012 \mathrm{G} 1$ & 0.8063 & 2.48 & $2.3 \times 10^{-20}$ & 0.82 & $1.43 \times 10^{-18}$ & $1.47 \times 10^{-18}$ \\
\hline $\mathrm{J} 213748+0012$ & 1.0437 & 2.37 & $3.7 \times 10^{-20}$ & 0.78 & $2.19 \times 10^{-18}$ & $2.14 \times 10^{-18}$ \\
\hline $\mathrm{J} 213748+0012 \mathrm{G} 2$ & 1.1890 & 2.57 & $2.4 \times 10^{-20}$ & 0.75 & $1.54 \times 10^{-18}$ & $1.45 \times 10^{-18}$ \\
\hline $\mathrm{J} 213748+0012 \mathrm{G} 3$ & 1.2144 & 2.43 & $2.4 \times 10^{-20}$ & 0.76 & $1.45 \times 10^{-18}$ & $1.39 \times 10^{-18}$ \\
\hline $\mathrm{J} 215200+0625 \mathrm{G} 1$ & 1.0534 & 2.28 & $2.1 \times 10^{-20}$ & 0.67 & $1.19 \times 10^{-18}$ & $1.01 \times 10^{-18}$ \\
\hline $\mathrm{J} 215200+0625$ & 1.1761 & 2.60 & $1.7 \times 10^{-20}$ & 0.66 & $1.10 \times 10^{-18}$ & $9.14 \times 10^{-19}$ \\
\hline $\mathrm{J} 215200+0625 G 2$ & 1.3190 & 2.41 & $3.6 \times 10^{-20}$ & 0.66 & $2.17 \times 10^{-18}$ & $1.79 \times 10^{-18}$ \\
\hline $\mathrm{J} 215200+0625 \mathrm{G} 3$ & 1.4309 & 2.60 & $2.1 \times 10^{-20}$ & 0.66 & $1.36 \times 10^{-18}$ & $1.13 \times 10^{-18}$ \\
\hline
\end{tabular}

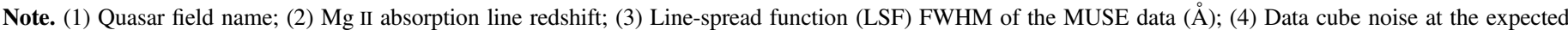

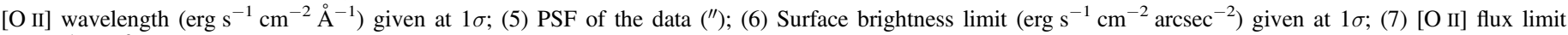
$\left(\mathrm{erg} \mathrm{s}^{-1} \mathrm{~cm}^{-2}\right)$ given at $1 \sigma$.

following relation:

$$
\mathrm{AB}=-2.5 \log _{10}(f)-5 \log _{10}(\langle\lambda\rangle)-2.406
$$

where $f$ is the flux in $\operatorname{erg~s}^{-1} \mathrm{~cm}^{-2} \AA^{-1}$ and $\langle\lambda\rangle$ the filter central wavelength in $\AA$.

The comparison between MUSE and SDSS magnitudes is shown in Table 2. For both fields (J2137+0012 and J2152 $+0625)$, the agreement is around $1 / 10$ of a magnitude. In addition, another data reduction was performed using CubeFix and CubeSharp (S. Cantalupo 2016, in preparation) in order to show cleaner images of the fields in the Appendix (Figures 14 and 15).

\subsubsection{UVES Observation and Reduction}

The high-resolution spectra for J213748+0012 and J215200 +0625 were taken with UVES mounted on the $8.2 \mathrm{~m}$ VLT at Paranal, Chile (Dekker et al. 2000). These two fields were observed in DDT time under the program 293.A-5038(A). UVES is a cross-dispersed echelle spectrograph with two arms, which are functionally identical: one covers the wavelengths in the range 3000-5000 $\AA$ (Blue) and the other covers the range $4200-11000 \AA$ (Red). The details of the observational campaigns are presented in Table 3 . The slit width of 1.2 arcsec and a CCD readout with $2 \times 2$ binning used for all the observations resulted in a spectral resolution power $\mathrm{R} \approx 38000$ dispersed on pixels of $\sim 1.3 \mathrm{~km} \mathrm{~s}^{-1}$. The settings were chosen in order to have a maximum of absorptions from host galaxies (from Fe II $\lambda 2586$ to $\mathrm{Mg} \mathrm{I}$ 22852). The Common Pipeline Language (version 6.3) of the UVES pipeline was used to bias correct and flat-field the exposures and then to extract the wavelength and flux calibrated spectra. After the standard reduction, the custom software UVES popler ${ }^{14}$ (version 0.66) was used to combine the extracted echelle orders into single 1D spectra. The continuum was fitted with low-order polynomials.

\section{MEGAFLOW SAMPLE FIRST RESULTS}

\subsection{Galaxy Detections}

As mentioned, the two fields (SDSS J213748+0012 and SDSS J215200+0625) were selected to each have at least three absorbing systems with $W_{r}>0.5 \AA$ (see Table 4).

In each MUSE field, we search for [O II] $\lambda \lambda 3727,3729$ emission lines corresponding to the $\mathrm{Mg}$ II absorption redshifts

\footnotetext{
${ }^{14}$ http://astronomy.swin.edu.au/ mmurphy/UVES_popler/
}

seen in the quasar spectrum. However, the MUSE field of view of $1^{\prime} \times 1^{\prime}$ allows us to search for other companions in the fields, giving insight into the environment related to the host. We allow the potential host galaxies to have a redshift difference within a velocity interval of $\approx 1000 \mathrm{~km} \mathrm{~s}^{-1}$ with respect to the absorber redshift $\left(z_{\mathrm{gal}}=z_{\mathrm{abs}} \pm 0.01\right.$ for a $z \approx 1$ galaxy). This velocity interval is set to prevent selection effects on surrounding gas velocities and thus not rejecting gas, which is able to escape the gravitational well of the host galaxy in case of outflowing gas (more details on escape velocity in Section 4.2). In the cases where there are multiple galaxy candidates for a single $\mathrm{Mg}$ II line, we select the galaxy with the smallest impact parameter from the quasar LOS. Table 4 shows the detection rates for each field. For one of the undetected galaxies, the expected emission line falls near a sky emission line at $7618 \AA$ (the $z \approx 1.0437$ absorber in SDSS J213748 $+0012)$ and the other line is too faint to be detected. For the reader interested in all of the galaxies detected in these MUSE data, we provide in the appendix a catalog with all the galaxies for which a redshift could be determined.

We detect galaxies at redshifts of three of the four $\mathrm{Mg}$ II absorbers for the SDSS J213748+0012 quasar field (see Table 4). For the $\mathrm{Mg}$ II absorber at $z=0.8063$, we find one [O II] emission-line galaxy at a distance $b$ of $88 \mathrm{kpc}$. For the $z=1.1890 \mathrm{Mg}$ II absorber, we also find one galaxy at an impact parameter of $63 \mathrm{kpc}$. For the last $z=1.2144 \mathrm{Mg}$ II absorber, we find three [O II] emitters, at impact parameters of 87,212 , and $246 \mathrm{kpc}$. Given the large impact parameters of the latter two galaxies compared to the typical galaxy halo at these redshifts, and given the large $\mathrm{Mg}$ II REW of $1 \AA$, we assume the galaxy with the smallest impact parameter to be the host galaxy.

For the SDSS J215200+0625 field, we also detect galaxies at the redshifts of three out of the four Mg II absorbers (see Table 4). Two galaxies are identified for the first $\mathrm{Mg}$ II absorber at $z=1.0534$, at impact parameters of 45 and $189 \mathrm{kpc}$. The host of the second absorber at $z=1.1761$ is not detected in spite of the wavelength for the expected [O II] line being clear of $\mathrm{OH}$ lines. The third $\mathrm{Mg}$ II absorption has a redshift of 1.3190 and has only one galaxy corresponding to that redshift at an impact parameter of $34 \mathrm{kpc}$. The last $\mathrm{Mg}$ II absorption is at $z=1.4309$ and we found four [O II] emitters at that redshift, which have impact parameters of 62, 78, 184, and $211 \mathrm{kpc}$ (see Figure 7) and might be indicative of a group environment. Among these four, two have impact parameters very close to each other (62 and $78 \mathrm{kpc}$ ). We choose to assume that the 
Table 6

Morphokinematics Results on Host Galaxies

\begin{tabular}{|c|c|c|c|c|c|c|c|c|c|c|}
\hline $\begin{array}{l}\text { Galaxy } \\
\text { (1) }\end{array}$ & $\begin{array}{l}z_{\text {abs }} \\
\text { (2) }\end{array}$ & $\begin{array}{l}z_{\text {gal }} \\
(3)\end{array}$ & $\begin{array}{c}b \\
(4)\end{array}$ & $\begin{array}{c}S / N \\
(5)\end{array}$ & $\begin{array}{c}\text { Size } \\
(6)\end{array}$ & $\begin{array}{c}i \\
(7)\end{array}$ & $\begin{array}{c}V_{\max } \\
(8)\end{array}$ & $\begin{array}{l}\text { Flux } \\
(9)\end{array}$ & $\begin{array}{c}\alpha \\
(10)\end{array}$ & $\begin{array}{l}\text { Class } \\
\text { (11) }\end{array}$ \\
\hline $\mathrm{J} 213748+0012 \mathrm{G} 1$ & 0.8063 & $0.80690 \pm 0.00001$ & $88.1 \pm 0.2$ & 11 & $2.43 \pm 0.06$ & $49.6 \pm 1.4$ & $126.2 \pm 5$ & $8.67 \times 10^{-17}$ & $25 \pm 1$ & Inflow \\
\hline $\mathrm{J} 213748+0012 \mathrm{G} 2$ & 1.1890 & $1.18925 \pm 0.00001$ & $63.7 \pm 0.2$ & 11 & $3.15 \pm 0.08$ & $55.6 \pm 0.8$ & $15.9 \pm 8$ & $1.47 \times 10^{-16}$ & $\ldots$ & Merger \\
\hline $\mathrm{J} 213748+0012 \mathrm{G} 3$ & 1.2144 & $1.21397 \pm 0.00003$ & $87.2 \pm 0.2$ & 4.5 & $5.38 \pm 0.33$ & $40.4 \pm 5.0$ & $166.5 \pm 18$ & $4.18 \times 10^{-17}$ & $47 \pm 2$ & Ambig. \\
\hline J215200+0625G1 & 1.0534 & $1.05335 \pm 0.00001$ & $45.4 \pm 0.2$ & 9.3 & $5.52 \pm 0.09$ & $69.4 \pm 0.7$ & $161.4 \pm 2$ & $1.09 \times 10^{-16}$ & $4 \pm 1$ & Inflow \\
\hline $\mathrm{J} 215200+0625 \mathrm{G} 2$ & 1.3190 & $1.31843 \pm 0.00005$ & $34.0 \pm 0.2$ & 4.2 & $3.06 \pm 0.51$ & $58.9 \pm 10.8$ & $130.6 \pm 29$ & $1.99 \times 10^{-17}$ & $88 \pm 5$ & Wind \\
\hline $\mathrm{J} 215200+0625 \mathrm{G} 3$ & 1.4309 & $1.43033 \pm 0.00004$ & $62.5 \pm 0.2$ & 10.5 & $1.51 \pm 0.12$ & $13.3 \pm 3.4$ & $298.5 \pm 39$ & $5.05 \times 10^{-17}$ & $72 \pm 20$ & Wind/Ambig. \\
\hline
\end{tabular}

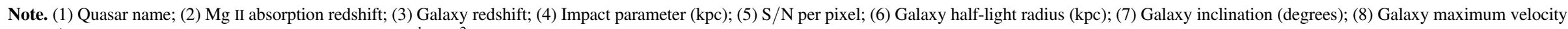
$\left(\mathrm{km} \mathrm{s}^{-1}\right)$; (9) Integrated [O II] flux of the galaxy (erg s${ }^{-1} \mathrm{~cm}^{-2}$ ); (10) Azimuthal angle (degrees); (11) Class (inflow-pair/wind-pair) based on $\alpha$ selection. 


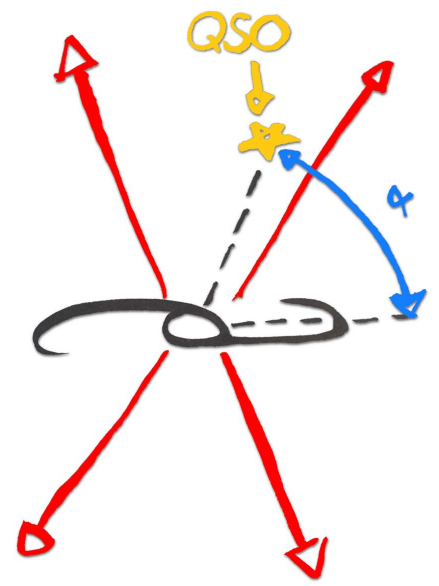

Figure 1. Scheme representing the azimuthal angle: the galaxy is represented at the center in black, the red arrows represent the outflowing gas expelled from both side of the galaxy minor axis. The azimuthal angle $\alpha$ is represented by the blue angle between the galaxy major axis and the quasar LOS (in yellow).

closest galaxy (at $62 \mathrm{kpc}$ ) should be responsible for the $\mathrm{Mg}$ II absorption, because it is the most massive and the brightest $\left(V_{\max }=298 \quad\right.$ and $200 \mathrm{~km} \mathrm{~s}^{-1}, \quad$ [O II] fluxes being $5.05 \times 10^{-17} \mathrm{erg} \mathrm{s}^{-1} \mathrm{~cm}^{-2}$ and $1.38 \times 10^{-17} \mathrm{erg} \mathrm{s}^{-1} \mathrm{~cm}^{-2}$, respectively).

Using the propagated noise in the MUSE data cube, we can estimate flux (and surface brightness) limits on the expected [O II] emission line for the non-detected host galaxies. For the SDSS J213748+0012 quasar field, at the first expected [O II] wavelength $(\sim 6730 \AA)$, with a noise of $2.3 \times 10^{-20}$ $\operatorname{erg~s}^{-1} \mathrm{~cm}^{-2} \AA^{-1}(1 \sigma)$, we estimate a surface brightness limit of $1.43 \times 10^{-18} \mathrm{erg} \mathrm{s}^{-1} \mathrm{~cm}^{-2} \operatorname{arcsec}^{-2}(1 \sigma)$ for emission-line objects (assuming a $\mathrm{FWHM}=2.48 \AA$ ). This corresponds to a flux limit of $1.04 \times 10^{-18} \mathrm{erg} \mathrm{s}^{-1} \mathrm{~cm}^{-2}(1 \sigma)$ for an unresolved emitter at 0 ". 82 seeing. The flux limit is $\sqrt{2}$ times for the [O II] doublet (assuming a resolved doublet), or $1.47 \times 10^{-18}$ $\mathrm{erg} \mathrm{s}^{-1} \mathrm{~cm}^{-2}(1 \sigma)$, which corresponds to an SFR of $0.13 M_{\odot} \mathrm{yr}^{-1}$ at $z=1$, typical of our sample. Surface brightness and flux limits are shown in Table 5.

\subsection{SFR Determination}

We use the $L_{\mathrm{O} \text { II }}(\lambda \lambda 3727,3729)$ luminosity to estimate the SFR as follows. We use the Kennicutt (1998) calibration, which assumes a Salpeter (1955) initial mass function (IMF):

$$
\operatorname{SFR}\left(M_{\odot} \mathrm{yr}^{-1}\right)=(1.4 \pm 0.4) \times 10^{-41} L([\mathrm{O} \mathrm{II}])_{\mathrm{o}}\left(\mathrm{erg} \mathrm{s}^{-1}\right)
$$

where $L([\mathrm{O} \mathrm{II}])_{\mathrm{o}}$ is the $[\mathrm{O} \mathrm{II}]$ observed luminosity. Using a Chabrier (2003) IMF and assuming a mean flux attenuation of $A_{V}=1$, which is typical for $z=1$ galaxies (e.g., Charlot et al. 2002), gives the same results (within 10\%) as Equation (2).

Equation (4) (Kewley et al. 2004, hereafter K04) also uses a Salpeter IMF, but makes no assumption of reddening. In their paper, they show that using the "average" attenuation correction of 0.3 mag leads to underestimation of the high SFR[O II] $\left(>1 M_{\odot} \mathrm{yr}^{-1}\right)$ and overestimation of the low SFRs. They provide a way of deriving the $E(B-V)$ (Equations (16) and (18) of K04) color excess, which leads to a more accurate mean attenuation, assuming that $A_{V}=3.1 \times E(B-V)$. We choose to use the following equations (Equations (3) and (4) from K04) to derive our SFRs.

$$
\begin{gathered}
\operatorname{SFR}\left(M_{\odot} \mathrm{yr}^{-1}\right)=(6.58 \pm 1.65) \times 10^{-42} L([\mathrm{O} \mathrm{II}])_{\mathrm{i}}\left(\mathrm{erg} \mathrm{s}^{-1}\right) \\
L([\mathrm{O} \mathrm{II}])_{\mathrm{i}}=3.11 \times 10^{-20} L([\mathrm{O} \mathrm{II}])_{\mathrm{o}}^{1.495}
\end{gathered}
$$

\subsection{Galaxy Morphokinematic Properties}

Before classifying the galaxy-quasar pairs as favorable for gas outflows or inflows based on the azimuthal angle $\alpha$ of the apparent quasar location with respect to the galaxy major axis, we need to determine the galaxy's major-axis position angle (PA). ${ }^{15}$

We determine the PAs from the morphokinematic properties of each galaxy, using two approaches. First, we used the 2D fitting tool $\mathrm{Camel}^{16}$ on the [O II] emission lines to extract velocity and dispersion maps as in Epinat et al. (2012) in order to establish whether the galaxy has a regular velocity field compatible with a disk. Second, we use the GalPaK ${ }^{3 \mathrm{D}}$ algorithm (Bouché et al. 2015a, 2015b) to derive simultaneously the morphological and kinematic properties of these galaxies using the continuum-subtracted subcubes extracted around the $[\mathrm{OII}]$ emission lines. $\mathrm{GalPaK}^{3 \mathrm{D} 17}$ uses a disk parametric model with 10 free parameters and a Monte Carlo Markov Chain algorithm with nontraditional sampling laws in order to efficiently probe the parameter space. Because the algorithm uses a 3D kernel to convolve the model with the spatial PSF or seeing, and the instrument LSF, it returns the intrinsic (free of the PSF) galaxy properties (such as half-light radius, inclination, and maximum velocity). Other parameters include the major-axis PA, the galaxy flux, position, redshift, and intrinsic velocity dispersion. Results on the geometrical and kinematic properties of each galaxy are presented in Table 6.

Figures 2-7 show GalPaK ${ }^{3 \mathrm{D}}$ reconstructed models as well as Camel velocity maps for the six galaxies in the two fields. In Figure 2 (SDSS J213748+0012 field), the other emission sources are the quasar and a star's residual continuum. In these figures, the left panel corresponds to a narrow-band image of 30 pixels $(37.5 \AA)$ around the galaxy's [O II] emission lines. The background continuum has been subtracted, so that we can only see the galaxy in emission. In each of these Figures, we see the galaxy (inside the white rectangles) within $15^{\prime \prime}$ of the quasar LOS (represented by a white cross). In the two right columns of these Figures, [O II] integrated flux and velocity maps are shown. The top row corresponds to a $2 \times 2$ ( 2 pixels FWHM) spatial Gaussian-smoothed flux map (left) and the Camel velocity map (right). The bottom row shows the $\mathrm{GalPaK}^{3 \mathrm{D}}$ model flux (left) and the PSF-deconvolved velocity (right) maps. We can see that in all cases, except in Figure 3 for the dispersion-dominated SDSS J213748+0012G2 galaxy, the model flux maps from $\mathrm{GalPaK}^{3 \mathrm{D}}$ are in a good agreement with the observed flux, and that $\mathrm{GalPaK}^{3 \mathrm{D}}$ and Camel velocity maps are consistent. Table 6 lists the resulting parameters for each galaxy.

$\mathrm{GalPaK}^{3 \mathrm{D}}$ results are reliable if the central galaxy pixel has, at minimum, an $\mathrm{S} / \mathrm{N}$ pixel $^{-1}$ of 3 (Bouché et al. 2015). For each galaxy, we have $\mathrm{S} / \mathrm{N}$ pixel $^{-1}$ of $11.0,11.0,4.5,9.3,4.2$, and 10.5 for SDSS J213748+0012G1, G2, G3 and SDSS $\mathrm{J} 215200+0625 \mathrm{G} 1, \mathrm{G} 2$ and G3, respectively. We checked that

\footnotetext{
15 The PA of a galaxy is the angle between the galaxy major axis and the celestial north.

${ }^{16}$ The source code can be found at https://bitbucket.org/bepinat/camel.git.

17 The source code can be found at http://galpak.irap.omp.eu.
} 

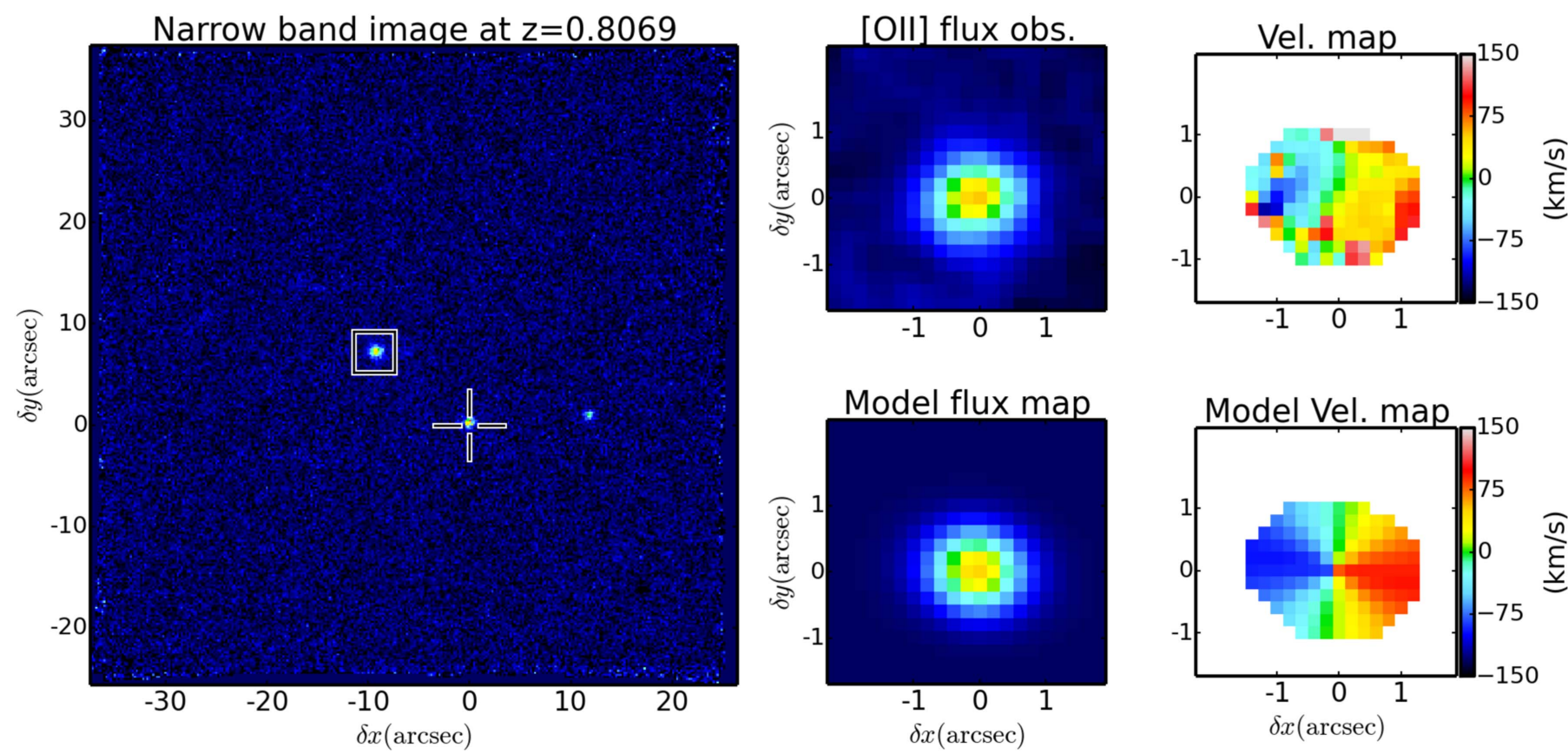

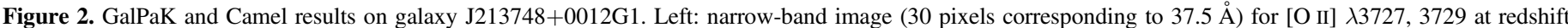

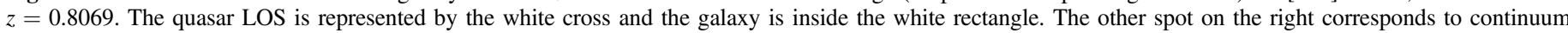

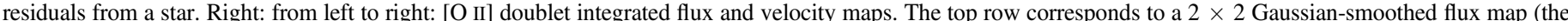

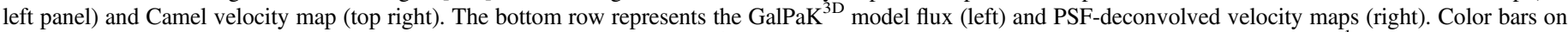
the right show the velocities of the corresponding velocity maps, in $\mathrm{km} \mathrm{s}^{-1}$. This galaxy has a maximum signal-to-noise ratio $(\mathrm{S} / \mathrm{N})$ pixel ${ }^{-1}$ of $\approx 11$.
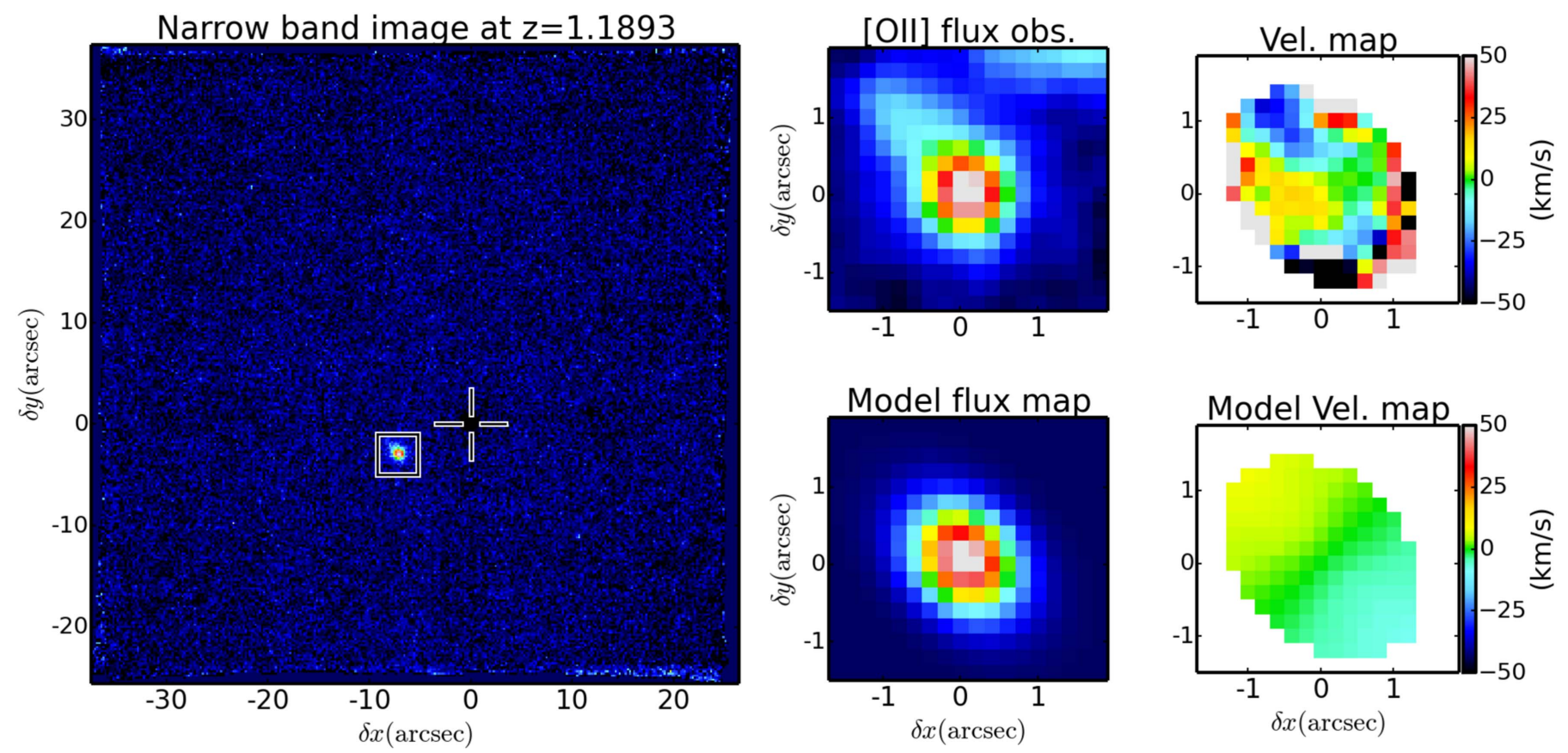

Figure 3. Same as Figure 2, but for $\mathrm{J} 213748+0012 \mathrm{G} 2$ at redshift $z=1.1893$. This galaxy has a maximum $\mathrm{S} / \mathrm{N}$ pixel ${ }^{-1}$ of $\approx 11$. For this galaxy, we can see that the velocity maps do not agree with each other. Because one part of the galaxy is not reproduced by our model and clearly has a flux component (top middle panel), this galaxy seems to be a merger and, therefore, the azimuthal angle of this pair is not reliable.

the parameters have converged for each galaxy as well as crosschecked on raw data.

\subsection{Classification and Notes on the Individual Cases}

To put constraints on galactic outflows, we first need to select galaxy-quasar pairs suitable for wind studies (wind- pairs). To do so, we measure the angle between the galaxy major axis and the apparent quasar location, which is referred to as the azimuthal angle $\alpha$ (see Figure 1). Depending on this angle, the quasar LOS is likely to probe different phenomena around the galaxy. If $55^{\circ} \leqslant \alpha \leqslant 90^{\circ}$, the quasar's position on the sky is roughly along the galaxy minor axis and is likely to cross the outflowing material of 

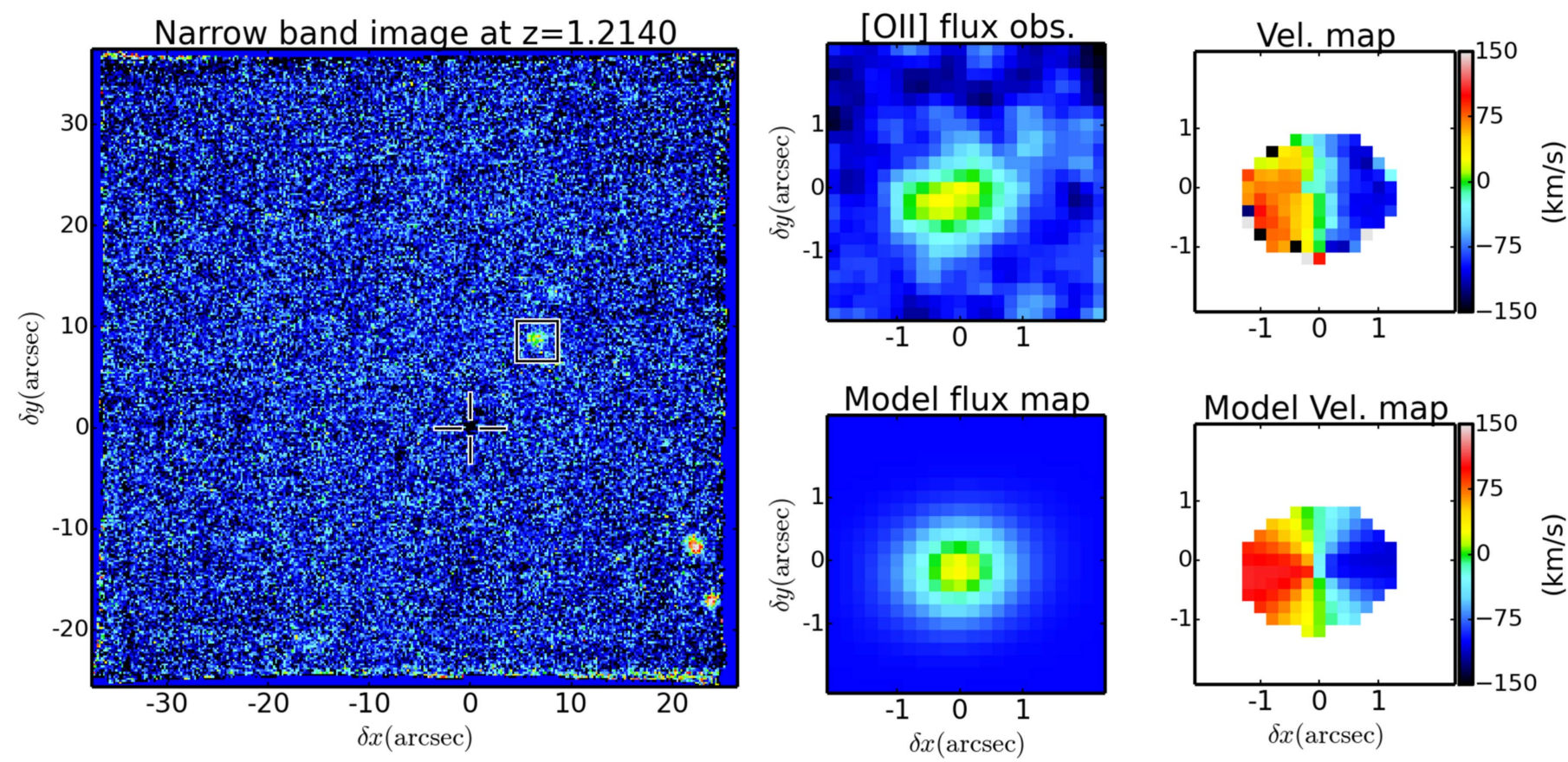

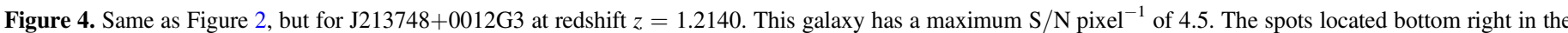

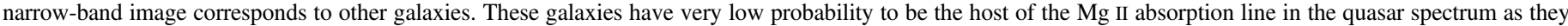
are located further away from the quasar LOS (212 and $246 \mathrm{kpc}$ ).
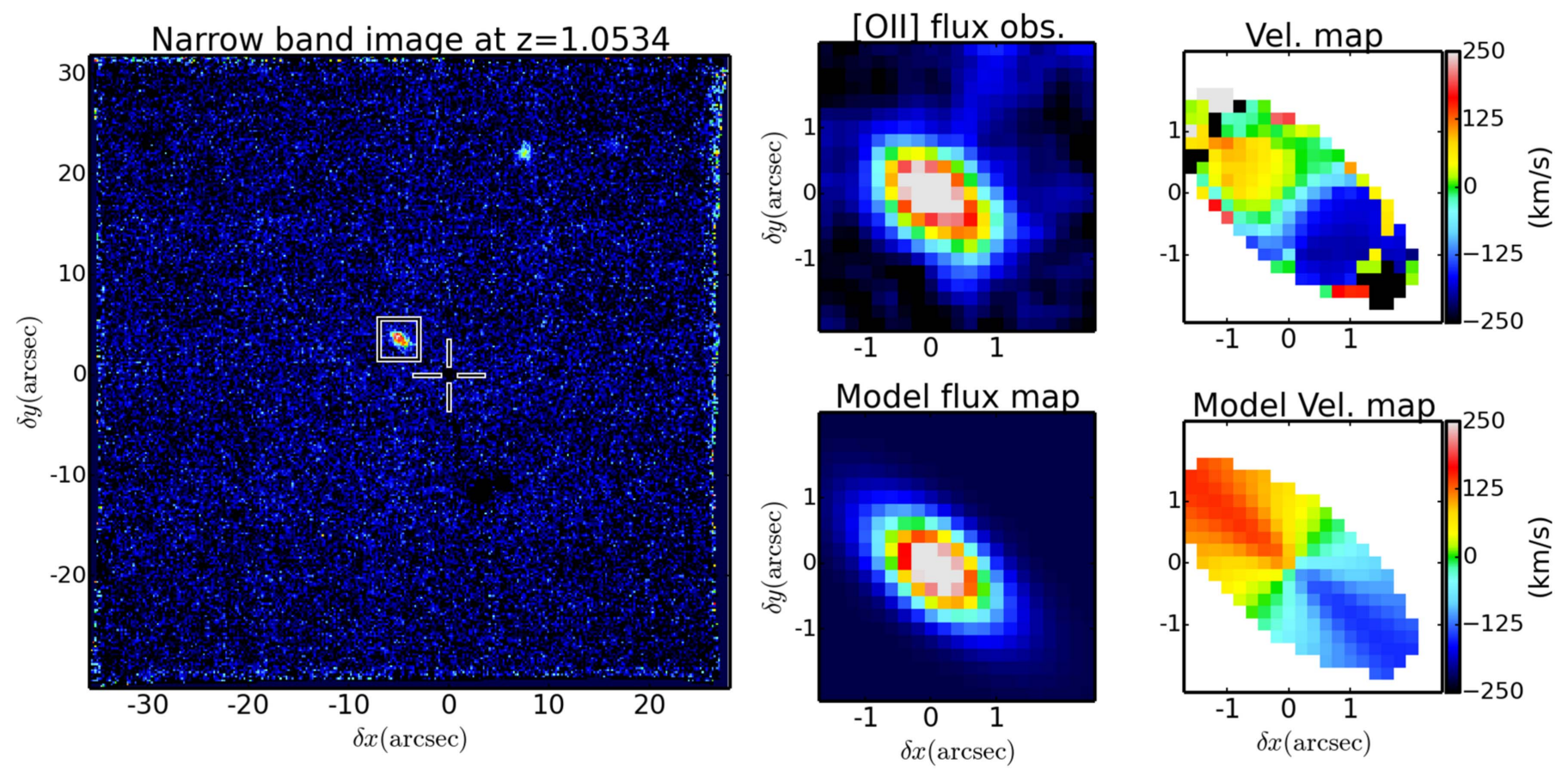

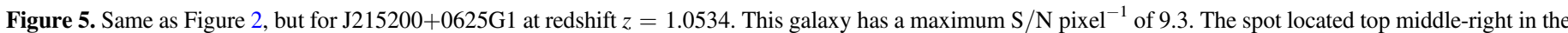

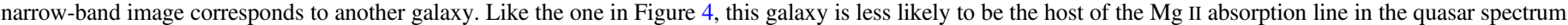
as it is located further away from the quasar LOS (189 kpc).

the galaxy ${ }^{18}$ (e.g., Bordoloi et al. 2011, 2014; Kacprzak et al. 2012, 2014). If a pair has such an azimuthal angle, it will be classified as a wind-pair. On the other hand, if the quasar is positioned along the galaxy major axis $\left(0^{\circ} \leqslant \alpha \leqslant 30^{\circ}\right)$, the quasar LOS is likely to probe inflowing or circumgalactic gas. With such a configuration, we classify

\footnotetext{
18 The Bordoloi papers have the definition of azimuthal angle reversed, i.e., their minor axis correspond to an $\alpha$ angle $<45^{\circ}$.
}

the pair as suitable for accretion studies (inflow-pair). In between, $\left(35^{\circ} \leqslant \alpha \leqslant 55^{\circ}\right)$, we cannot distinguish between these two extreme cases.

In addition to the azimuthal angle, if a galaxy has a low inclination, classification can be ambiguous given that the uncertainty on the PA will be large. Figure 8 shows galaxy inclination as a function of quasar azimuthal angle. From the five detected galaxies in the two quasar fields that are non-mergers, two are classified as inflow-pairs, one is an ambiguous case as its 

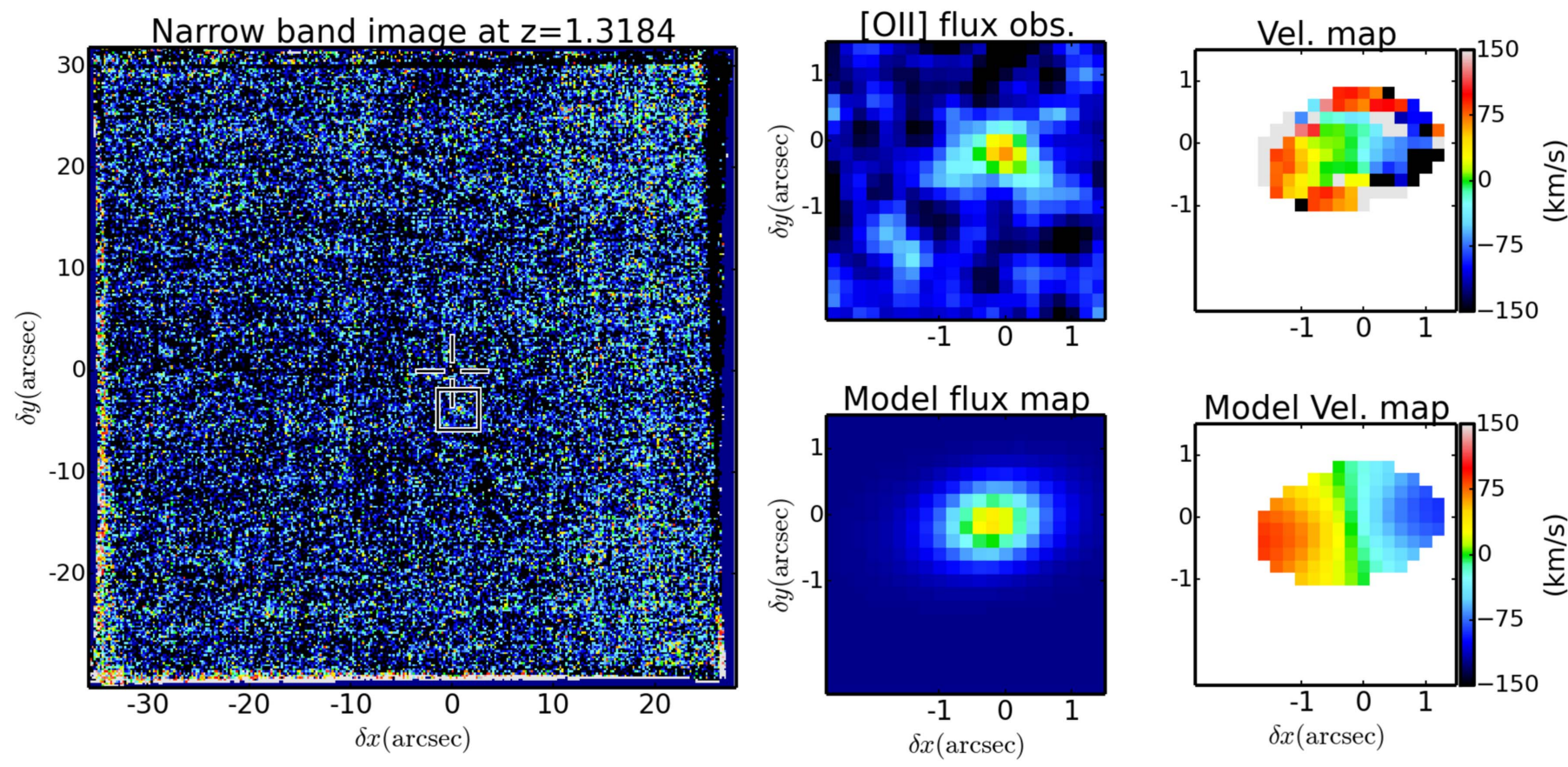

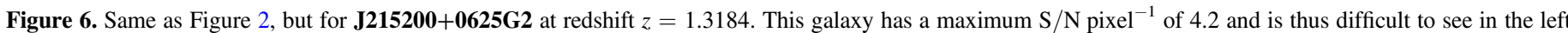
image, but can be seen in the smoothed [O II] flux image.

azimuthal angle is $47^{\circ}$, one is a face-on galaxy and only 1 $(\mathbf{J} 215200+0625 G 2)$ can be robustly classified as a wind-pair.

\subsubsection{SDSS J213748+0012G1 Galaxy}

The first detected galaxy ("G1") in the SDSS J213748+0012 quasar field (Figure 2) has an impact parameter $b \approx 88 \mathrm{kpc}$ and corresponds to the $z_{\text {abs }} \approx 0.8063 \mathrm{Mg}$ II absorption lines with a REW $W_{r}^{\lambda 2796}$ of $0.789 \AA$. This J213748+0012G1 galaxy is inclined by $i \approx 49 \pm 1.4^{\circ}$ and its derived maximum rotation velocity is $V_{\max } \approx 127 \pm 5 \mathrm{~km} \mathrm{~s}^{-1}$. With an [O II] integrated flux of $8.7 \times 10^{-17} \mathrm{erg} \mathrm{s}^{-1} \mathrm{~cm}^{-2}$, its SFR is $\approx 6.3 \pm 0.7 M_{\odot} \mathrm{yr}^{-1}$. In Figure 2, it can be seen that the morphology and the PA are well reproduced by $\mathrm{GalPaK}^{3 \mathrm{D}}$. The azimuthal angle $\alpha$ with the quasar LOS is $\alpha=25$ deg, i.e., the LOS is aligned with the major axis.

\subsubsection{SDSS J213748+0012G2 Galaxy}

The galaxy J213748+0012G2 (Figure 3) corresponding to the $z_{\text {abs }} \approx 1.1890 \mathrm{Mg}$ II absorption lines with a REW $W_{r}^{\lambda 2796}$ of $0.308 \AA$ in the $\mathrm{J} 213748+0012$ quasar spectrum, has an impact parameter of $b \approx 64 \mathrm{kpc}$ and a total [O II] doublet flux of $1.47 \times 10^{-16} \mathrm{erg} \mathrm{s}^{-1} \mathrm{~cm}^{-2}$. From the [O II] integrated flux we derive an SFR of $\approx 41 \pm 8.0 M_{\odot} \mathrm{yr}^{-1}$. This galaxy has a large velocity dispersion $\sigma \approx 114 \pm 2.3 \mathrm{~km} \mathrm{~s}^{-1}$, i.e., it is a dispersion-dominated system with $V / \sigma \sim 0.2$. Furthermore, the velocity field derived from the line-fitting algorithm Camel does not agree with its morphology, i.e., its morphological and kinematic main axes are strongly misaligned, by $\approx 80^{\circ}$ (Figure 3). This is a strong indication for a merger, and therefore, this galaxy will not be considered as a wind case, since the PA of this galaxy is ambiguous.

\subsubsection{SDSS J213748+0012G3 Galaxy}

The other galaxy (J213748+0012G3, Figure 4) from the $\mathrm{J} 213748+0012$ field corresponding to the $\mathrm{Mg}$ II absorption lines at redshift $z_{\mathrm{abs}} \approx 1.2144$ and a REW $W_{r}^{\lambda 2796}$ of $1.144 \AA$ has an impact parameter $b$ of $\approx 87 \mathrm{kpc}$. This galaxy has an inclination $i \approx 40 \pm 5^{\circ}$, a maximum rotational velocity $V_{\max } \approx 166 \pm 18 \mathrm{~km} \mathrm{~s}^{-1}$, and an [O II] flux of $4.17 \times$ $10^{-17} \mathrm{erg} \mathrm{s}^{-1} \mathrm{~cm}^{-2}$. From this flux, we derive an SFR of $\approx 8.9 \pm 1.1 M_{\odot} \mathrm{yr}^{-1}$. In contrast to $\mathrm{J} 213748+0012 \mathrm{G} 2$, the kinematic and morphological PAs agree well (Figure 4), hence the $3 \mathrm{D} \mathrm{GalPaK}{ }^{3 \mathrm{D}}$ model accounts for the $3 \mathrm{D}$ emission of this galaxy. In this case, the quasar LOS is at $\approx 45^{\circ}$ from the major axis of this galaxy, and this pair is thus classified as ambiguous.

\subsubsection{SDSS J215200+0625G1 Galaxy}

The first detected galaxy from the SDSS J215200+0625 quasar field corresponds to the $\mathrm{Mg}$ II absorption lines at redshift $z_{\text {abs }} \sim 1.0534$ with a REW $W_{r}^{\lambda 2796}$ of $0.545 \AA$. This galaxy $(\mathrm{J} 215200+0625 \mathrm{G} 1)$ has an impact parameter $b \approx 45 \mathrm{kpc}$, a maximum rotational velocity $V_{\max } \approx 161 \pm 2 \mathrm{~km} \mathrm{~s}^{-1}$, and an inclination $i \approx 69^{\circ} \pm 0.7$. With an [O II] integrated flux of $1.09 \times 10^{-16}$ we derive an SFR of $\approx 19.0 \pm 3.1 M_{\odot} \mathrm{yr}^{-1}$. For this galaxy, Figure 5 shows a good agreement between the GalPaK $^{3 \mathrm{D}}$ and Camel flux and velocity maps. We can clearly see that the quasar LOS is aligned with the major axis of this galaxy with $\alpha=4 \mathrm{deg}$, and is thus classified as an inflow-pair.

\subsubsection{SDSS J215200+0625G2 Galaxy}

The galaxy $(\mathrm{J} 215200+0625 \mathrm{G} 2)^{19}$ corresponding to the redshift $z_{\text {abs }} \approx 1.3190 \mathrm{Mg}$ II absorption lines with a REW $W_{r}^{\lambda 2796}$ of $1.424 \AA$ has an impact parameter $b \approx 34 \mathrm{kpc}$. The derived galaxy redshift is 1.31845 with an inclination of $i \approx 59 \pm 11^{\circ}$ and a maximum rotational velocity $V_{\max } \approx 130 \pm 29 \mathrm{~km} \mathrm{~s}^{-1}$. With an [O II] flux of $\approx 1.99 \times 10^{-17} \mathrm{erg} \mathrm{s}^{-1} \mathrm{~cm}^{-2}$, we derive an SFR of $\approx 4.6 \pm 0.4 M_{\odot} \mathrm{yr}^{-1}$. Even if this galaxy is faint, as can be seen in Figure 6, its $\mathrm{GalPaK}^{3 \mathrm{D}}$-derived morphology and PA are in

\footnotetext{
${ }^{19}$ In the paper (text, tables and figures), the only wind-pair will appear in bold font to help the reader.
} 

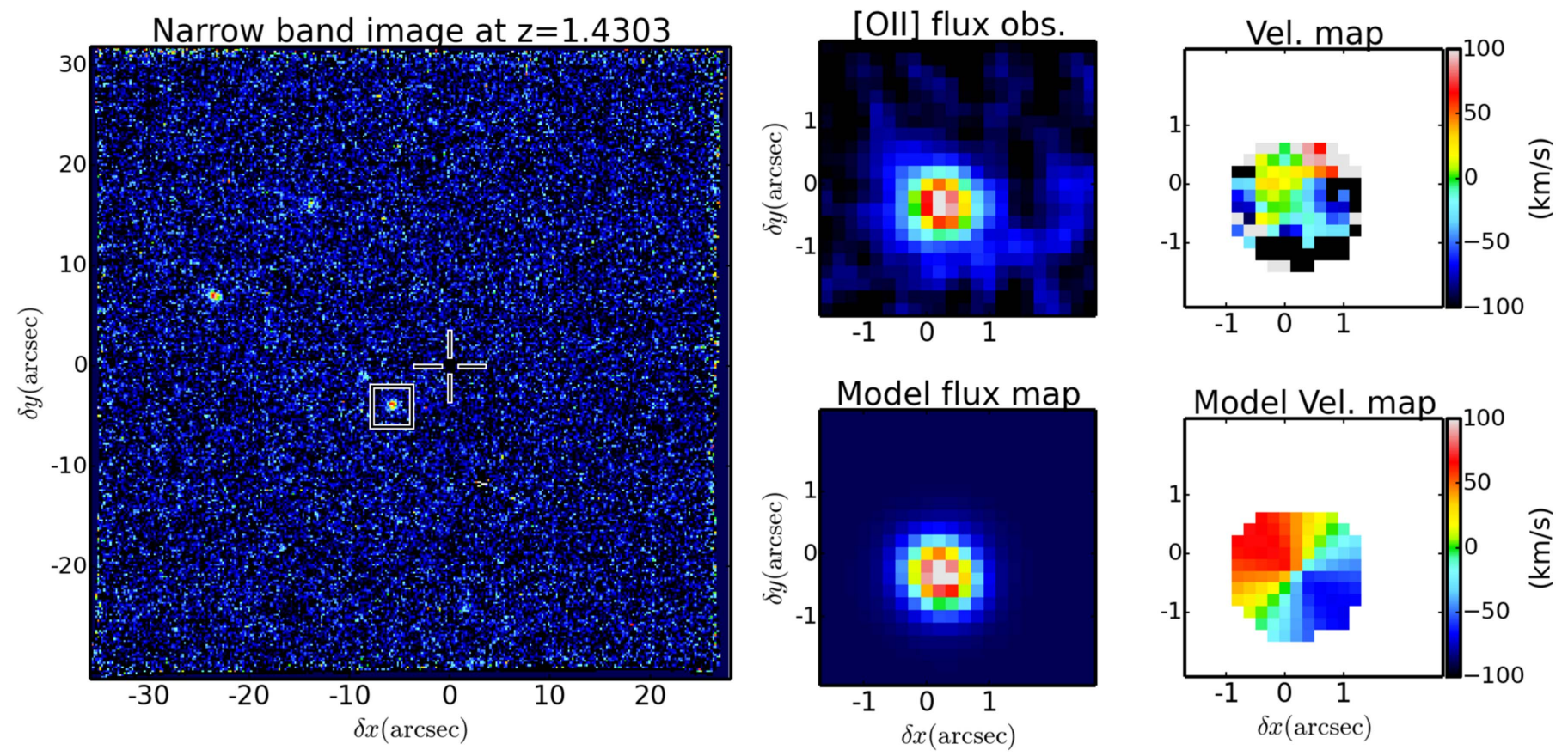

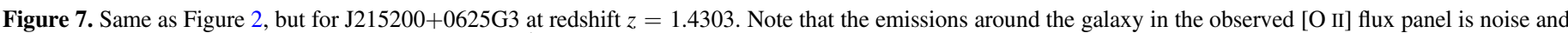

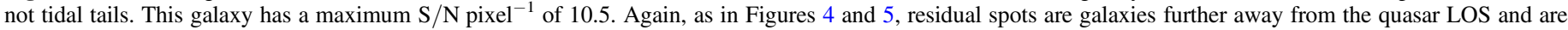

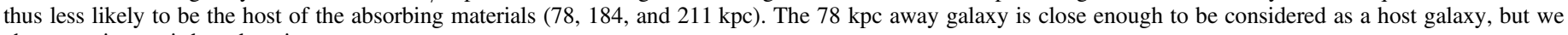
choose to ignore it based on impact parameter argument.

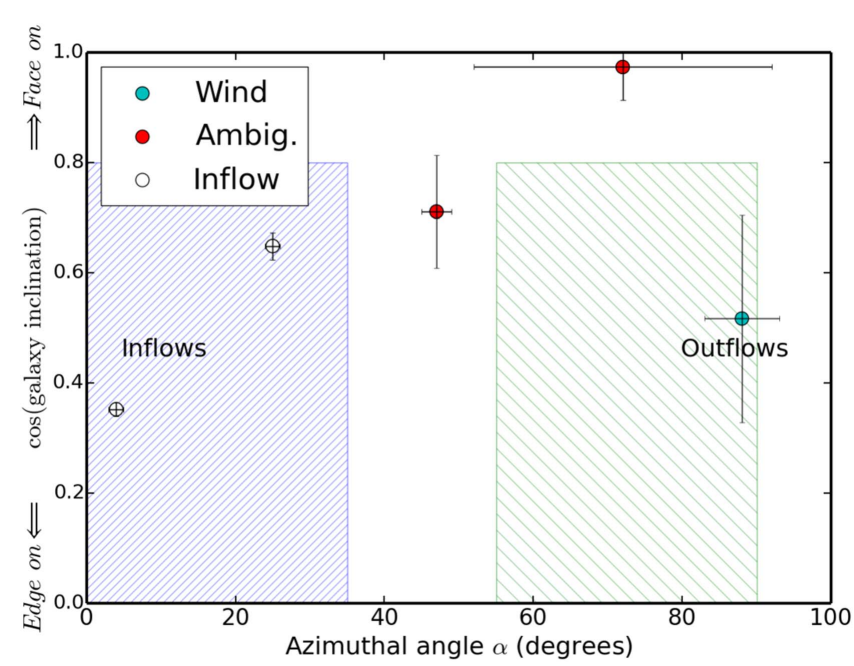

Figure 8. Galaxy inclinations as a function of azimuthal angle $\alpha$ for the five nonmerger galaxies detected in the two fields $\mathrm{J} 213748+0012$ and $\mathrm{J} 215200$ +0625 . We note that only one galaxy is classified as a wind-pair. The dashed areas correspond to azimuthal angle ranges for which we classify pairs as inflow-pairs (blue and narrow dashes) or wind-pairs (green and wider dashes). These areas stop for face-on galaxies, as uncertainties on position angles are too large and thus make it difficult to classify pairs.

good agreement with Camel maps. The quasar LOS is aligned with the minor axis of this galaxy with $\alpha=88 \pm 5 \mathrm{deg}$.

\subsubsection{SDSS J215200+0625G3 Galaxy}

The last galaxy $(\mathrm{J} 215200+0625 \mathrm{G} 3)$ in the $\mathrm{J} 215200+0625$ quasar field has an impact parameter $b \approx 63 \mathrm{kpc}$ and corresponds to the $\mathrm{Mg}$ II absorption lines at redshift $z_{\text {abs }} \approx 1.4309$ with $W_{r}^{\lambda 2796}=1.152 \AA$. The galaxy has an inclination of $i \approx 13 \pm 4^{\circ}$, a maximum rotational velocity $V_{\max } \approx 298 \pm 40 \mathrm{~km} \mathrm{~s}^{-1}$, and an [O II] integrated flux of $\approx 5.05 \times 10^{-17} \mathrm{erg} \mathrm{s}^{-1} \mathrm{~cm}^{-2}$. With this flux, we derive an SFR of $\approx 19 \pm 3.0 M_{\odot} \mathrm{yr}^{-1}$. Figure 7 shows that the morphology is in agreement with Camel, but the PA derived for this galaxy is more uncertain due to the low inclination of this galaxy. With an azimuthal angle of $\alpha=72 \pm 20 \mathrm{deg}$ and its low inclination, we cannot determine whether the quasar LOS is aligned with the minor or major axis of the galaxy.

\subsection{Radial Dependence of CGM}

For each quasar spectrum, we measure the REW for the Mg II absorption lines $\left(W_{r}^{\lambda 2796}\right)$ in the UVES data and compare them with the SDSS values $W_{r}^{\lambda 2796}$ (see Table 7). We find that the results are consistent with each other. We also calculate REWs of the Mg II $\lambda 2803, \mathrm{Mg}$ I $\lambda 2852$, Fe II $\lambda 2586$, and Fe II $\lambda 2600$ in UVES quasar spectra. Results are shown in Table 7. Figures 9 and 10 show the UVES MgI $\lambda 2852, \mathrm{Mg}$ II $\lambda \lambda 2796,2802$, and Fe II $\lambda \lambda 2586,2600$ absorption profiles and label the measured REW of each profile for both quasar fields.

One of the first deductions we can make from Figures 9 and 10 is that there is no clear difference (like different asymmetry behavior for instance) between what seems to be outflowing material and circumgalactic or inflowing gas concerning the different absorption lines.

Figure 11 shows the distribution of REW $W_{\mathrm{r}}^{\lambda 2796}$ for pairs with an azimuthal angle $\alpha>45^{\circ}$ as a function of impact parameter $b$ for this work as well as Kacprzak et al. (2011a, 2011b) and Schroetter et al. (2015). This Figure shows that for wind-pairs, as mentioned in Bouché et al. (2012), we clearly see a tight correlation between $W_{\mathrm{r}}^{\lambda 2796}$ and $b$. This $W_{\mathrm{r}}^{\lambda 2796}-b$ correlation goes approximatively as $b^{-1}$. This figure shows that the anticorrelation between impact parameter $b$ and $W_{r}$ is again confirmed at $b<100 \mathrm{kpc}$. The scatter around the relation in Figure 11 is $\approx 0.3 \mathrm{dex}$ (delineated with the dotted lines). The 
SDSSJ213748 z=0.8069 Class:Inflow

(a)

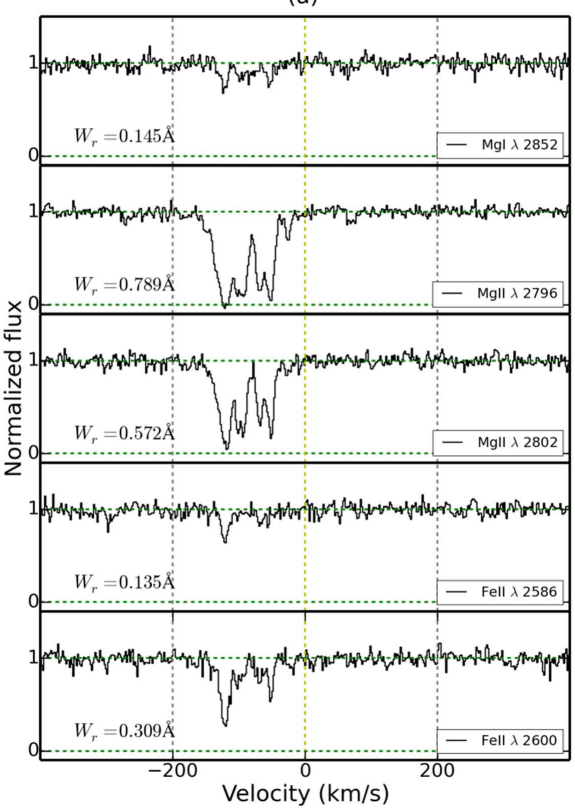

SDSSJ213748 z=1.1893 Class: Merger

(b)

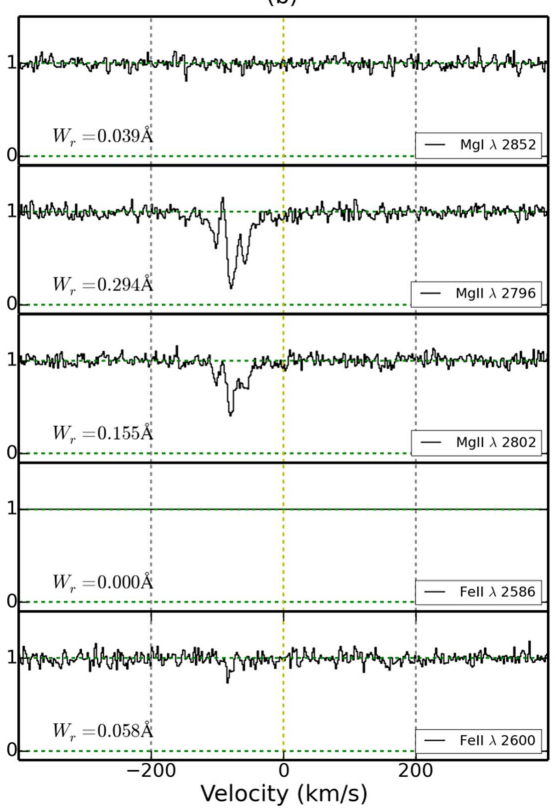

SDSSJ213748 z=1.2140 Class:Ambiguous

(c)

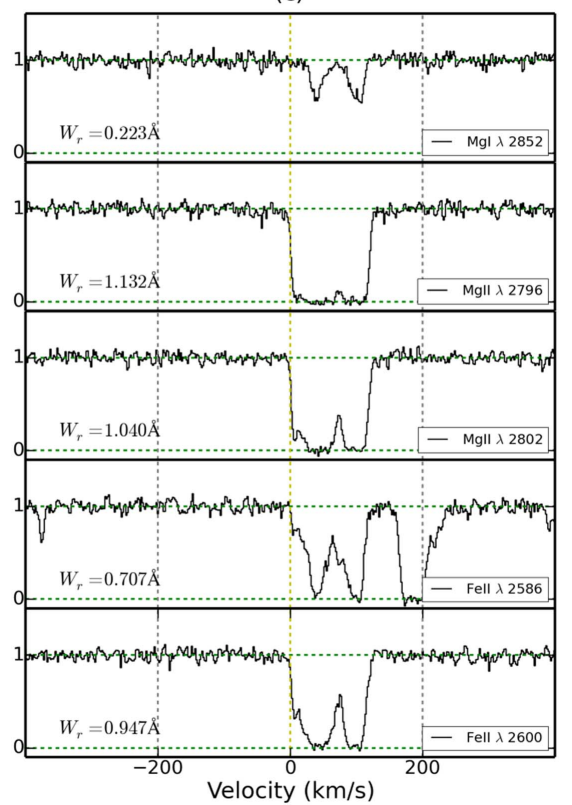

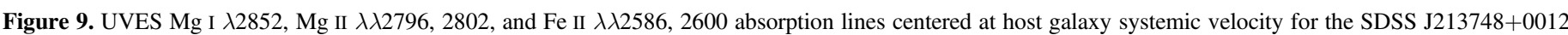

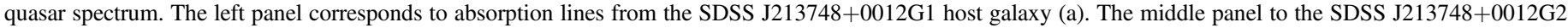

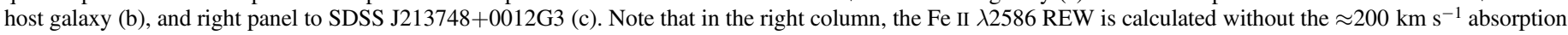
component.

Table 7

UVES Rest Equivalent Widths

\begin{tabular}{|c|c|c|c|c|c|c|c|c|}
\hline $\begin{array}{l}\text { Galaxy } \\
\text { (1) }\end{array}$ & $\begin{array}{c}W_{r}^{\lambda 2796}(\mathrm{SDSS}) \\
(2)\end{array}$ & $\begin{array}{l}W_{r}^{\lambda 2796} \\
\text { (3) }\end{array}$ & $\begin{array}{l}W_{r}^{\lambda 2802} \\
(4)\end{array}$ & $\begin{array}{l}W_{r}^{\lambda 2852} \\
\quad(5)\end{array}$ & $\begin{array}{l}W_{r}^{\lambda 2586} \\
\quad(6)\end{array}$ & $\begin{array}{l}W_{r}^{\lambda 2600} \\
\quad(7)\end{array}$ & $\begin{array}{c}\log \left(N_{\mathrm{H} i}\right) \\
(8)\end{array}$ & $\begin{array}{l}\text { Class } \\
(9)\end{array}$ \\
\hline $\mathrm{J} 213748+0012 \mathrm{G} 1$ & $0.724 \pm 0.09$ & $0.789 \pm 0.02$ & $0.572 \pm 0.02$ & $0.145 \pm 0.02$ & $0.135 \pm 0.02$ & $0.309 \pm 0.02$ & 19.24 & Inflow \\
\hline $\mathrm{J} 213748+0012 \mathrm{G} 2$ & $0.308 \pm 0.06$ & $0.294 \pm 0.02$ & $0.155 \pm 0.02$ & $0.039 \pm 0.02$ & $\cdots$ & $0.058 \pm 0.02$ & 18.61 & Merger \\
\hline $\mathrm{J} 213748+0012 \mathrm{G} 3$ & $1.122 \pm 0.06$ & $1.132 \pm 0.02$ & $1.040 \pm 0.02$ & $0.223 \pm 0.02$ & $0.707 \pm 0.02$ & $0.947 \pm 0.02$ & 19.58 & Ambig. \\
\hline $\mathrm{J} 215200+0625 \mathrm{G} 1$ & $0.522 \pm 0.14$ & $0.545 \pm 0.02$ & $0.460 \pm 0.02$ & $0.116 \pm 0.02$ & $0.175 \pm 0.02$ & $0.271 \pm 0.02$ & 19.01 & Inflow \\
\hline $\mathrm{J} 215200+0625 G 2$ & $1.347 \pm 0.12$ & $1.424 \pm 0.02$ & $1.065 \pm 0.02$ & $0.158 \pm 0.02$ & $0.322 \pm 0.02$ & $0.709 \pm 0.02$ & 19.71 & Wind \\
\hline $\mathrm{J} 215200+0625 \mathrm{G} 3$ & $1.152 \pm 0.11$ & $1.157 \pm 0.02$ & $\cdots$ & $\cdots$ & $0.122 \pm 0.02$ & $0.242 \pm 0.02$ & 19.59 & Wind/Ambig. \\
\hline
\end{tabular}

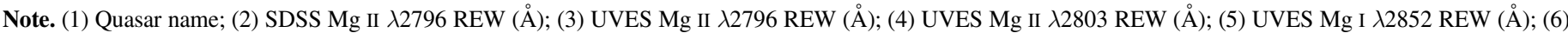
UVES Fe II $\lambda 2586$ REW ( $\AA$ ); (7) UVES Fe II $\lambda 2600$ REW ( $\AA$ ); (8) Gas column density at the impact parameter ( $\mathrm{cm}^{-2}$ ); (9) Class (inflow-pair/wind-pair) based on $\alpha$ selection.

solid line traces the fiducial $1 / b$ relation for mass-conserved biconical outflows (see Bouché et al. 2012).

\section{WIND MODEL}

In this section, we describe the wind modeling. We create a cone with an opening angle corresponding ${ }^{20}$ to $\theta_{\max }$ and fill it randomly with particles representing cold gas clouds being pushed away by a hot medium or radiation pressure. These particles are distributed such that their number goes like $1 / r^{2}$, where $r$ is the distance to the galaxy center. The particle density is normalized arbitrarily to reproduce the optical depth of the absorption profiles.

Such entrained clouds are accelerated to their terminal velocity quickly in a few $\mathrm{kpc}$ or $<10 \mathrm{kpc}$, since the pressure from the hot medium or the radiation field scales as $1 / r^{2}$. The range of impact parameters for the galaxy-quasar pair in our sample is always

\footnotetext{
${ }^{20} \theta_{\max }$ is defined from the central axis, and the cone subtends an area $\Omega$ of $\pi \cdot \theta_{\max }^{2}$
}

larger than $30 \mathrm{kpc}$. Hence, we assume, for simplicity, that the particles have a constant radial velocity corresponding to $V_{\text {out }}$. In addition, a single LOS probes a rather small range of distances from the host galaxy such that a gradient in the outflow velocity would have no significant impact on our results. So far, only in one LOS with an impact parameter less than $10 \mathrm{kpc}$ in Schroetter et al. (2015), did we require an accelerated wind profile.

We then orient the cone following the galaxy inclination and simulate the quasar LOS such that the galaxy-quasar pair matches the geometrical configuration of the MUSE data.

The particle velocities are then projected along the simulated quasar LOS and the distribution of the projected velocities gives us a simulated optical depth $\tau_{\mathrm{v}}$, which we turn into an absorption profile $\propto \exp \left(-\tau_{\mathrm{v}}\right)$. In order to facilitate comparison with the data, Poisson noise is added to the simulated absorption profile to simulate the instrumental noise. This noise is chosen to have the same level as the data.

The model has two main free parameters, the wind speed $V_{\text {out }}$ and $\theta_{\max }$ the wind opening angle. These two parameters 
(a)

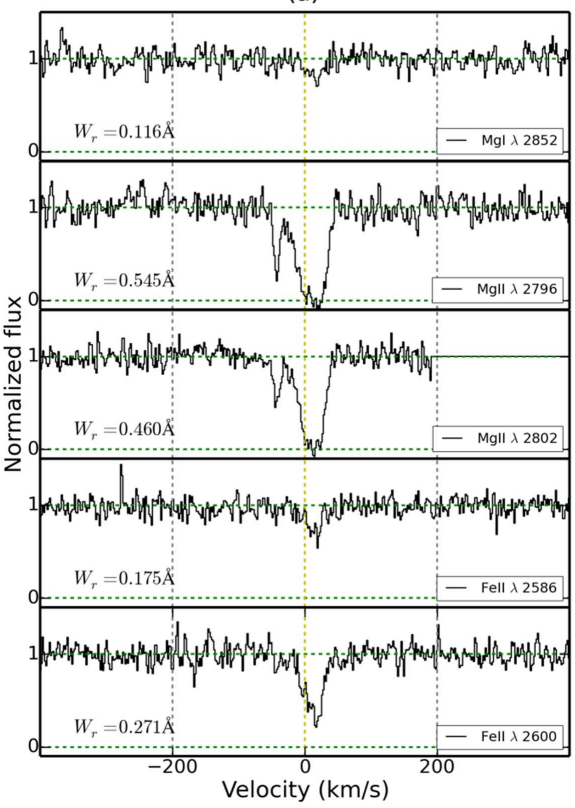

SDSSJ215200 $z=1.3185$ Class:Wind

(b)

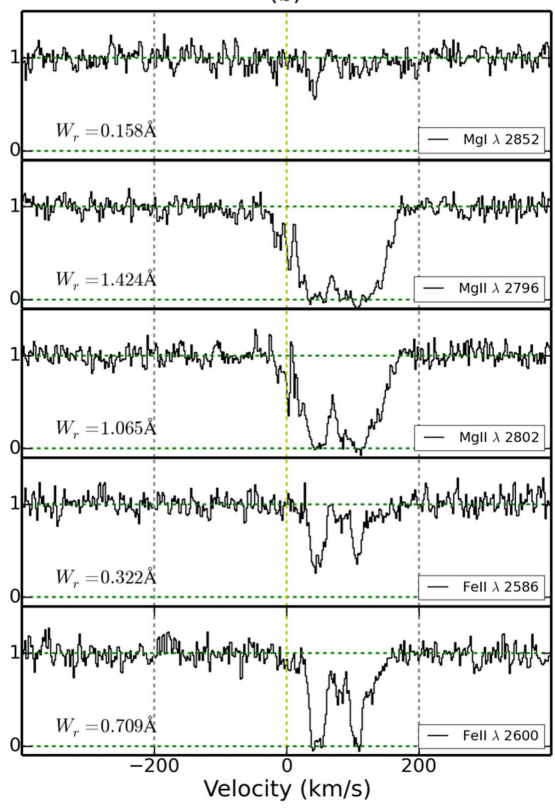

SDSSJ215200 z=1.4303 Class:Wind/Ambig.

(c)

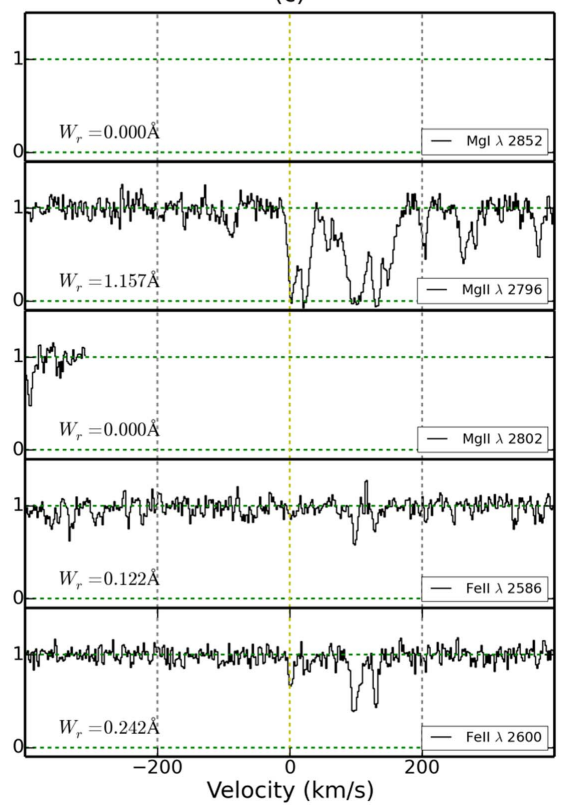

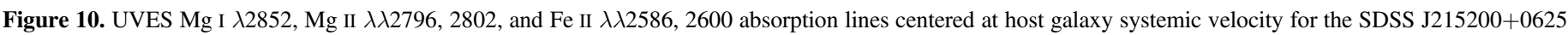

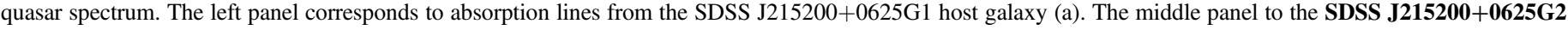
host galaxy (b), and right panel to SDSS J215200+0625G3 (c).

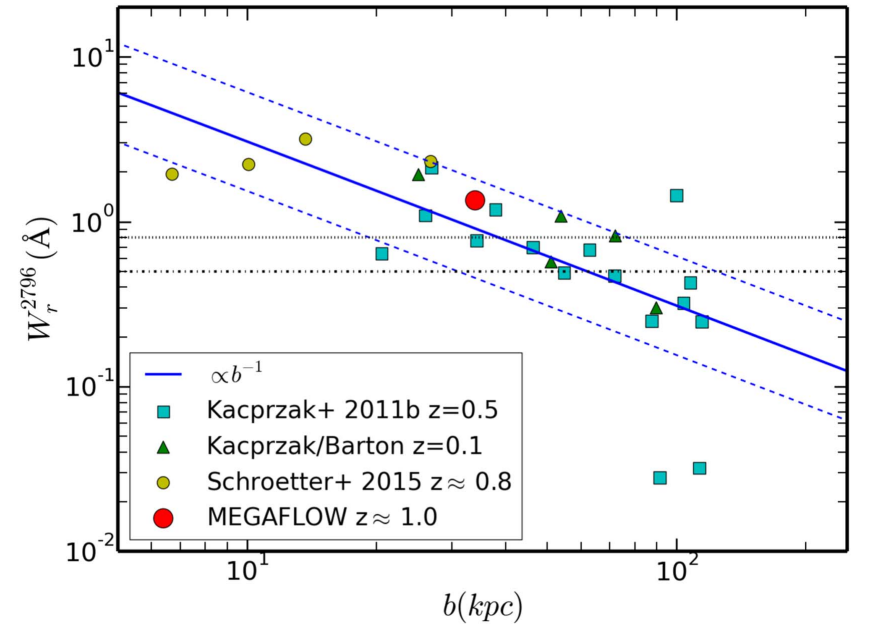

Figure 11. $W_{r}^{\lambda 2796}$ as a function of impact parameter $b$ for galaxy-quasar pairs classified as wind-pairs. The dashed blue lines show the 0.3 dex scatter. The horizontal dotted black lines represent the $W_{r}^{\lambda 2796}=0.8 \AA$ and $W_{r}^{\lambda 2796}=0.5 \AA$ selection limits.

are independent for a given galaxy inclination, as one can see from the following arguments (see also Schroetter et al. (2015) for more details). The outer edges of the absorption profile (reddest for a cone pointing away from the observer, bluest for a cone pointing toward the observer) depend directly on the wind velocity (Figure A-1 in Schroetter et al. 2015). The inner edge (toward Vsys) of the absorption profile depends directly on the wind opening angle $\theta_{\max }$ (Figure A-1 in Schroetter et al. 2015). Note that the galaxy inclination impacts the absorption profiles similarly to the $\theta_{\max }$ parameter, but since the inclination is determined by our 3D fit with $\mathrm{GalPaK}^{3 \mathrm{D}}$, there are no degeneracies.

In order to determine which model best reproduces the data, the best-fit model is visually found. However, given that there are stochastic features in the simulated profiles, we generate dozens of simulated profiles for a given set of parameters. The errors on these parameters are given by the range of values allowed by the data. We proceed as follows: We first generate models, changing only one parameter to fit one part of the absorption profile (outer part for $V_{\text {out }}$ or inner part for $\theta_{\max }$ ). Then, we change only the other parameter $\left(\theta_{\max }\right.$ or $\left.V_{\text {out }}\right)$ generating other models to fit the other part of the absorption. We generate models with a range of values of $10-500 \mathrm{~km} \mathrm{~s}^{-1}$ (with steps of $10 \mathrm{~km} \mathrm{~s}^{-1}$ ) for $V_{\text {out }}$ and $20^{\circ}-50^{\circ}$ (with steps of $5^{\circ}$ ) for $\theta_{\max }$. As mentioned before, with these two parameters being independent, there is no degeneracy between the generated models. We use these parameter ranges to fit the data, since outflows are likely to be collimated in a cone with an opening angle around $30^{\circ}$ (e.g., Chen et al. 2010; Bordoloi et al. 2011, 2014; Bouché et al. 2012; Kacprzak et al. 2012; Martin et al. 2012; Rubin et al. 2014)

Examples on how the wind model behaves, as we change the different parameters, can be seen in the appendix of Schroetter et al. (2015).

\subsection{The Wind-pair Case of J215200+0625G2}

Figure 10, middle column (b), shows the UVES Mg I $\lambda 2852$, Mg II $\lambda \lambda 2796,2802$, and Fe II $\lambda \lambda 2586,2600$ absorption lines for this galaxy-quasar pair. From this Figure, we can see that the $\mathrm{Mg}$ II $\lambda \lambda 2796,2802$ absorption lines are saturated and thus the need to simulate the absorption from Fe II $\lambda 2586$, which are the only nonsaturated absorption lines in the presented transitions.

The bottom right panel of Figure 12 shows the UVES Fe II $\lambda 2586$ absorption lines corresponding to the $\mathrm{J} 215200+0625 \mathrm{G} 2$ galaxy redshift of $z=1.3184$. This absorption is the one we intend to fit in order to constrain outflow properties, since other absorption lines like $\mathrm{Mg}$ II are saturated (see panel (b) of Figure 10). In this profile, we can see a suppression of 

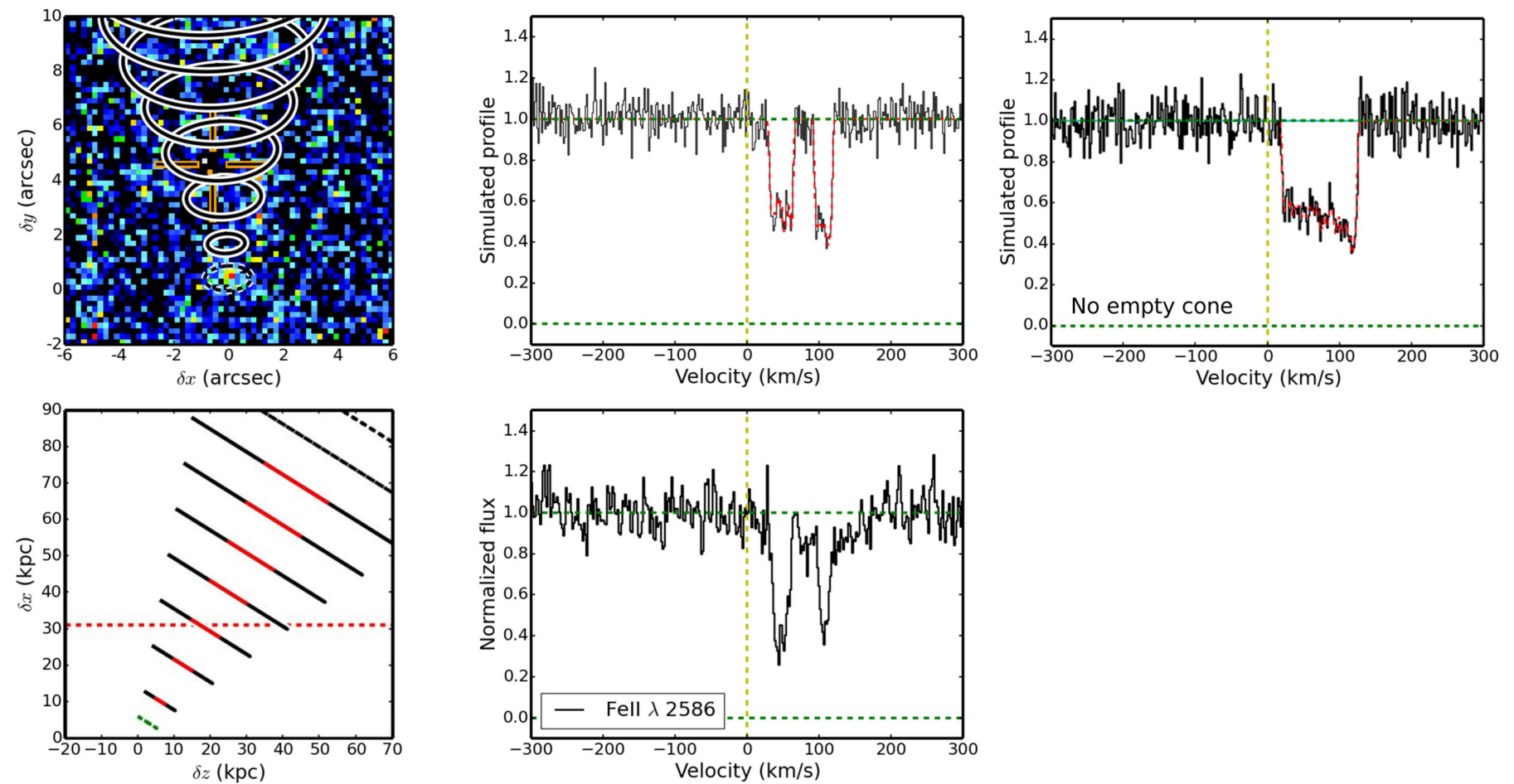

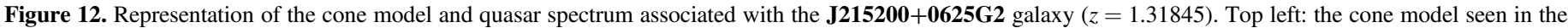

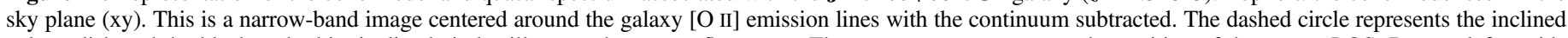

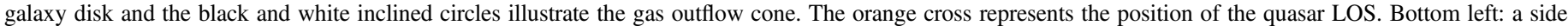

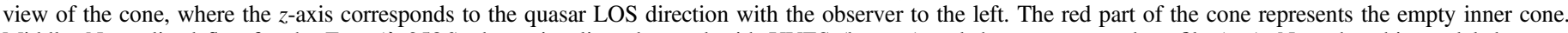

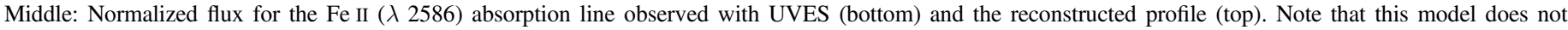

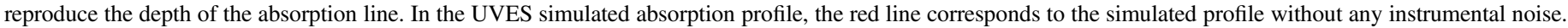

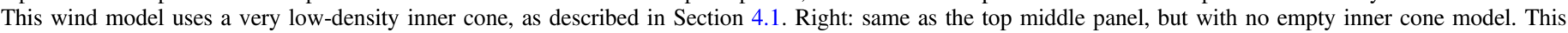

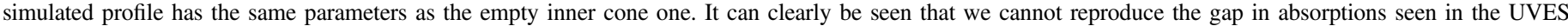

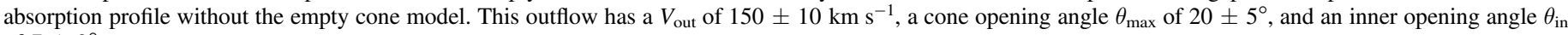
of $7 \pm 2^{\circ}$.

absorption around $80 \mathrm{~km} \mathrm{~s}^{-1}$. We first tried to fit this absorption with our wind model described in Section 4, but failed to reproduce this gap, even with stochastic effects. This lack of absorbing particles at these velocities shows that the outflowing cone must have a low-density region inside it.

Given that the geometry of this galaxy-quasar system (with a galaxy inclination $i$ of $59^{\circ}$ ) and that the quasar line of sight is crossing the outflowing cone near its middle $\left(\alpha=88^{\circ}\right)$, we thus developed a partially empty cone model in order to reproduce the absorption profile.

The principle is the same as in the wind model described in Section 4, except that we only fill the cone with particles from a certain opening angle $\theta_{\text {in }}$ to $\theta_{\max }$. The inner cone is thus empty. This model should only work if the azimuthal angle $\alpha$ of a galaxy-quasar system is above $\sim 80^{\circ}$, and thus the quasar LOS is crossing this empty region and creating a gap of velocities in the simulated profile.

This empty inner cone could be the signature of a hotter gas filling the inner cone, while the ionized gas traced by our lowionization lines would correspond to the walls of the outflowing cone in a manner similar to Fox et al. (2015) for the Milky Way and to Veilleux \& Rupke (2002) for NGC1482.

Figure 12 illustrates the resulting wind modeling for this galaxy. The first left column corresponds to the wind model representation. The top left panel shows a [O II] integrated flux, continuum-subtracted image with the orange cross showing the quasar LOS position. The inclined circles represent the outflowing cone. The bottom left panel represents a side view of the cone, with the quasar LOS being represented by the dashed red line, and with the observer on the left. This representation allows us to see if the outflowing material is ejected toward or away from us, assuming our cone model is representative. The red part of the cone represents the empty inner part.

In the middle column are represented the simulated profiles (top) and UVES spectrum around the absorption line Fe II $\lambda 2586$ (bottom). The red part of the simulated profile is the profile without instrumental noise and the apparent noise is due to stochastic effects from the Monte Carlo particle distribution. The red simulated absorption profile does not change much for the UVES data compared to the noise-added one. We also present in Figure 12, top right panel, a similar simulated profile (with the same parameters), but without the empty inner cone model. It can clearly be seen in this Figure that we cannot reproduce the gap shown in the data without an empty region.

The bottom middle panel corresponds to UVES data. It corresponds to the QSO spectrum absorption lines centered at the galaxy systemic velocity. The element Fe II $\lambda 2586$, corresponding to the absorption lines, is shown in the bottom middle column panel.

To reproduce the shape of this absorption profile and generate the simulated profile shown in the top middle panel of 
Table 8

Results for the Galaxy J215200+0625G2

\begin{tabular}{|c|c|c|c|c|c|c|c|c|c|}
\hline $\begin{array}{l}\text { Galaxy } \\
\text { (1) }\end{array}$ & $\begin{array}{l}b(\mathrm{kpc}) \\
\quad(2)\end{array}$ & $\begin{array}{c}\log \left(N_{\mathrm{H}}(b)\right) \\
\text { (3) }\end{array}$ & $\begin{array}{l}V_{\max } \\
(4)\end{array}$ & $\begin{array}{l}V_{\text {out }} \\
(5)\end{array}$ & $\begin{array}{l}\theta_{\max } \\
(6)\end{array}$ & $\begin{array}{l}\text { SFR } \\
\text { (7) }\end{array}$ & $\begin{array}{c}\dot{M}_{\text {out }} \\
(8)\end{array}$ & $\begin{array}{c}\frac{V_{\text {out }}}{V_{\text {esc }}} \\
(9)\end{array}$ & $\begin{array}{c}\eta \\
(10)\end{array}$ \\
\hline $\mathrm{J} 215200+0625 G 2$ & 34.0 & $19.7 \pm 0.07$ & $140.8 \pm 51$ & $150 \pm 10$ & $20 \pm 5.0$ & $4.6 \pm 0.4$ & $\begin{array}{l}1.7_{-0.8}^{+1.1} \\
1.1_{-0.6}^{+0.9}\end{array}$ & 0.52 & $\begin{array}{l}0.75 \\
0.49\end{array}$ \\
\hline
\end{tabular}

Note. (1) Galaxy name; (2) Impact parameter (kpc); (3) Gas column density at the impact parameter $\left(\mathrm{cm}^{-2}\right)$; (4) Maximum rotational velocity of the galaxy (km s ${ }^{-1}$ ); (5) Wind velocity $\left(\mathrm{km} \mathrm{s}^{-1}\right)$; (6) Cone opening angle (degrees) (7) SFR $\left(M_{\odot} \mathrm{yr}^{-1}\right)$; (8) Ejected mass rate for one cone $\left(M_{\odot} \mathrm{yr}^{-1}\right)$; (9) Ejection velocity divided by escape velocity; (10) Mass loading factor: ejected mass rate divided by SFR (for both cones). Values in the second row $\left(\dot{M}_{\text {out }}=1.1_{-0.6}^{+0.9} M_{\odot} \mathrm{yr}^{-1}\right.$ and $\left.\eta=0.49\right)$ correspond to the empty inner cone model.

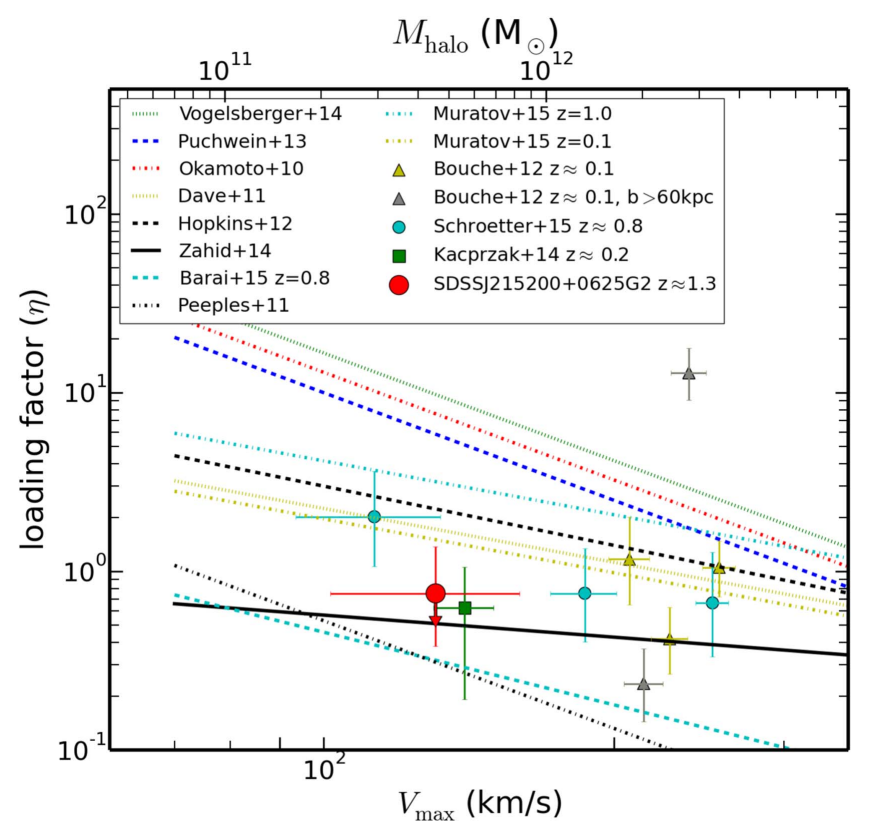

Figure 13. Comparison of mass loading factors assumed by theoretical/ empirical models (curves) with values derived from background quasar observations (dots and triangles) as a function of the maximum rotational velocity. The result from this work is represented by the red circle. The red arrow represents the loading factor of the SDSS J215200+0625G2 galaxy with the subtracted mass from the inner cone model. The cyan circles show the results for galaxies at $z \approx 0.8$ from Schroetter et al. (2015). The green square shows the mass loading factor for a $z \approx 0.2$ galaxy (Kacprzak et al. 2014). The triangles show the results for $z \approx 0.2$ galaxies from Bouché et al. (2012). The gray triangles show the galaxies with quasars located at $>60 \mathrm{kpc}$, where the mass loading factor is less reliable due to the large travel time needed for the outflow to cross the quasar LOS (several $100 \mathrm{Myr}$ ) compared to the short timescale of the $\mathrm{H} \alpha$-derived SFR ( 10Myr). The upper halo mass axis is scaled on $V_{\max }$ at redshift 0.8 from Mo \& White (2002).

Figure 12, we adjust the outflow speed $V_{\text {out }}$ and the cone opening angle $\theta_{\max }$, while keeping the geometrical parameters of the galaxy fixed, as described in Section 4.

The best values for reproducing the UVES Fe II $\lambda 2586$ absorption profile are an outflow velocity $V_{\text {out }}$ of $150 \pm 10 \mathrm{~km} \mathrm{~s}^{-1}$ and a cone opening angle $\theta_{\max }$ of $20^{\circ} \pm 5^{\circ}$. For this specific case, we derive an inner opening angle of the cone of $\theta_{\text {in }} \approx 7^{\circ}$.

\subsection{Outflow Rates}

Having constrained the outflow velocity and cone opening angle for the wind-pair, we can now derive the ejected mass rate $\dot{M}_{\text {out }}$ as well as the loading factor.

For our wind-pair, the equivalent width of the absorption lines only depends on $\theta_{\max }$ and $V_{\text {out }}$ (see Section 4). After
Table 9

MUSE Sources in the SDSS J213748+0012 Field with Redshifts

\begin{tabular}{|c|c|c|c|c|}
\hline ID & R.A. & Decl. & Redshift & Lines \\
\hline obj001 & $21: 37: 48.303$ & $+00: 12: 21.69$ & 0.132 & $\mathrm{O}$ III, $\mathrm{H}_{\beta}, \mathrm{H}_{\alpha}, \mathrm{N}$ II \\
\hline obj002 & $21: 37: 48.757$ & $+00: 12: 19.29$ & 0.156 & $\mathrm{O}$ III, $\mathrm{H}_{\beta}, \mathrm{H}_{\alpha}, \mathrm{N}$ II \\
\hline obj003 & $21: 37: 50.157$ & $+00: 12: 52.89$ & 0.315 & $\mathrm{H}_{\beta}, \mathrm{O}$ III \\
\hline obj004 & $21: 37: 48.370$ & $+00: 12: 23.89$ & 0.325 & $\begin{array}{c}\mathrm{O} \text { II, } \mathrm{O} \text { III, } \mathrm{H}_{\beta}, \\
\mathrm{H}_{\alpha}, \mathrm{N} \text { II }\end{array}$ \\
\hline obj005 & $21: 37: 48.370$ & $+00: 12: 24.09$ & 0.325 & $\mathrm{O}$ II, $\mathrm{O}$ III, $\mathrm{H}_{\beta}, \mathrm{H}_{\alpha}$ \\
\hline obj006 & $21: 37: 48.930$ & $+00: 12: 38.69$ & 0.409 & $\mathrm{O}$ II, $\mathrm{O}$ III, $\mathrm{H}_{\alpha}, \mathrm{N}$ II \\
\hline obj007 & $21: 37: 49.223$ & $+00: 12: 20.09$ & 0.410 & $\mathrm{O}$ II, O III, $\mathrm{H}_{\beta}$ \\
\hline obj008 & $21: 37: 49.810$ & $+00: 12: 15.69$ & 0.442 & $\mathrm{O}$ II \\
\hline obj009 & 21:37:48.477 & $+00: 12: 30.09$ & 0.543 & $\mathrm{O}$ II, $\mathrm{O}$ III, $\mathrm{H}_{\beta}$ \\
\hline obj010 & $21: 37: 48.450$ & $+00: 12: 29.49$ & 0.543 & $\mathrm{O}$ II, O III, $\mathrm{H}_{\beta}$ \\
\hline obj011 & $21: 37: 50.450$ & $+00: 12: 02.89$ & 0.580 & $\mathrm{O}$ III, $\mathrm{H}_{\beta}$ \\
\hline obj012 & $21: 37: 48.983$ & $+00: 12: 55.09$ & 0.616 & $\mathrm{O}$ II, O III, $\mathrm{H}_{\beta}$ \\
\hline obj013 & $21: 37: 49.343$ & $+00: 12: 52.09$ & 0.684 & $\mathrm{O}$ II, O III, $\mathrm{H}_{\beta}$ \\
\hline obj014 & $21: 37: 47.743$ & $+00: 12: 46.69$ & 0.711 & $\mathrm{O}$ II \\
\hline obj015 & $21: 37: 49.530$ & $+00: 12: 14.69$ & 0.766 & O II \\
\hline obj016 & 21:37:48.317 & $+00: 12: 15.69$ & 0.767 & O II \\
\hline obj017 & $21: 37: 49.463$ & $+00: 12: 16.49$ & 0.767 & O II, O III \\
\hline obj018 & $21: 37: 49.023$ & $+00: 12: 27.29$ & 0.806 & $\mathrm{O}$ II, O III, $\mathrm{H}_{\beta}$ \\
\hline obj019 & $21: 37: 48.823$ & $+00: 12: 27.49$ & 0.806 & O II, O III \\
\hline obj020 & $21: 37: 50.157$ & $+00: 12: 30.89$ & 0.806 & O II, O III \\
\hline obj021 & $21: 37: 49.490$ & $+00: 12: 33.69$ & $\cdots$ & 8281.3 \\
\hline obj022 & $21: 37: 50.103$ & $+00: 12: 53.29$ & $\cdots$ & 6823. \\
\hline obj023 & $21: 37: 49.117$ & $+00: 12: 11.89$ & $\cdots$ & 6897. \\
\hline obj024 & $21: 37: 47.663$ & $+00: 12: 12.69$ & 0.900 & $\mathrm{O}$ II \\
\hline obj025 & $21: 37: 48.930$ & $+00: 12: 09.49$ & 0.902 & $\mathrm{O}$ II? \\
\hline obj026 & $21: 37: 48.517$ & $+00: 12: 05.69$ & $\cdots$ & 7079.69 \\
\hline obj027 & $21: 37: 48.063$ & $+00: 12: 33.69$ & $\ldots$ & 7376.81 \\
\hline obj028 & $21: 37: 48.437$ & $+00: 12: 46.29$ & 1.010 & O II \\
\hline obj029 & $21: 37: 48.837$ & $+00: 12: 42.69$ & 1.010 & O II \\
\hline obj030 & $21: 37: 48.970$ & $+00: 12: 09.49$ & 1.045 & O II \\
\hline obj031 & $21: 37: 49.970$ & $+00: 12: 09.09$ & 1.044 & O II \\
\hline obj032 & $21: 37: 49.970$ & $+00: 12: 15.29$ & 1.122 & $\mathrm{O}$ II \\
\hline obj033 & $21: 37: 48.903$ & $+00: 12: 17.69$ & 1.188 & O II \\
\hline obj034 & $21: 37: 46.837$ & $+00: 12: 02.89$ & 1.212 & O II \\
\hline obj035 & $21: 37: 47.970$ & $+00: 12: 29.09$ & 1.213 & $\mathrm{O}$ II \\
\hline obj036 & $21: 37: 46.943$ & $+00: 12: 08.89$ & 1.214 & O II \\
\hline obj037 & $21: 37: 47.850$ & $+00: 12: 33.49$ & 1.214 & $\mathrm{O}$ II \\
\hline obj038 & $21: 37: 50.410$ & $+00: 12: 20.09$ & 1.257 & O II \\
\hline obj039 & $21: 37: 48.370$ & $+00: 12: 04.69$ & 1.300 & $\mathrm{O}$ II \\
\hline obj040 & $21: 37: 47.717$ & $+00: 12: 46.89$ & $\cdots$ & 8569.12 \\
\hline obj041 & $21: 37: 48.730$ & $+00: 12: 15.29$ & 5.941 & 8434.53 Ly $\alpha ?$ \\
\hline obj042 & $21: 37: 48.823$ & $+00: 12: 27.49$ & 6.442 & 9043.03 Ly $\alpha ?$ \\
\hline
\end{tabular}

Note. Within these 42 emitters, 36 have identified emission lines.

testing several opening angles and outflow velocities, we fitted the width of the absorption profile created by gas outflowing from the galaxy. The asymmetry of the profile depends on the 
Table 10

MUSE Sources in the SDSS J215200+0625 Field with Redshifts

\begin{tabular}{|c|c|c|c|c|}
\hline$\overline{\mathrm{ID}}$ & R.A. & Decl. & Redshift & Lines \\
\hline obj001 & 21:52:02.018 & $+06: 25: 47.66$ & 0.433 & $\mathrm{O}$ II, O III, $\mathrm{H}_{\beta}$ \\
\hline obj002 & $21: 52: 02.246$ & $+06: 25: 25.06$ & 0.439 & $\mathrm{O}$ II \\
\hline obj003 & 21:51:58.905 & $+06: 25: 20.26$ & 0.452 & $\mathrm{O}$ II, O III, $\mathrm{H}_{\beta}$ \\
\hline obj004 & 21:52:02.085 & $+06: 25: 13.26$ & 0.489 & $\mathrm{O}$ II, O III, $\mathrm{H}_{\beta}$ \\
\hline obj005 & 21:51:58.409 & $+06: 24: 54.86$ & 0.517 & O II \\
\hline obj006 & $21: 51: 59.429$ & $+06: 25: 43.06$ & 0.554 & O II, O III \\
\hline obj007 & 21:52:02.273 & $+06: 24: 56.06$ & 0.597 & $\mathrm{O}$ II, O III, $\mathrm{H}_{\beta}$ \\
\hline obj008 & $21: 52: 00.770$ & $+06: 25: 17.26$ & $3.931 ?$ & 5992.37 Ly $\alpha ?$ \\
\hline obj009 & 21:51:59.200 & $+06: 24: 54.86$ & $4.196 ?$ & 6314.05 Ly $\alpha ?$ \\
\hline obj010 & $21: 51: 58.878$ & $+06: 25: 01.46$ & 0.742 & $\mathrm{O}$ II, O III, $\mathrm{H}_{\beta}$ \\
\hline obj011 & 21:51:59.912 & $+06: 25: 15.66$ & 0.748 & $\mathrm{O}$ II, $\mathrm{H}_{\beta}$ \\
\hline obj012 & 21:52:02.139 & $+06: 25: 31.26$ & 0.770 & $\mathrm{O}$ II, $\mathrm{O}$ III, $\mathrm{H}_{\beta}$ \\
\hline obj013 & $21: 51: 59.375$ & $+06: 25: 40.26$ & 0.786 & $\mathrm{O}$ II \\
\hline obj014 & 21:52:00.341 & $+06: 25: 22.46$ & 0.332 & $\mathrm{O}$ II, O III, $\mathrm{H}_{\alpha}$ \\
\hline obj015 & 21:52:01.092 & $+06: 25: 16.26$ & 0.824 & O II, O III \\
\hline obj016 & 21:52:00.636 & $+06: 25: 37.66$ & 0.289 & $\mathrm{H}_{\alpha}, \mathrm{N}$ II \\
\hline obj017 & 21:51:58.597 & $+06: 25: 11.86$ & 0.847 & O II? \\
\hline obj018 & 21:51:59.818 & $+06: 25: 29.66$ & 0.873 & O II \\
\hline obj019 & 21:52:00.126 & $+06: 25: 13.06$ & 0.879 & O II, O III \\
\hline obj020 & 21:52:00.234 & $+06: 24: 50.86$ & 0.438 & $\mathrm{O}$ II, O III, $\mathrm{H}_{\beta}$ \\
\hline obj021 & 21:51:59.630 & $+06: 25: 40.46$ & 0.943 & O II \\
\hline obj022 & 21:52:00.287 & $+06: 25: 06.46$ & 0.989 & $\mathrm{O}$ II \\
\hline obj023 & 21:52:02.058 & $+06: 25: 40.46$ & 1.013 & $\mathrm{O}$ II \\
\hline obj024 & $21: 51: 58.436$ & $+06: 25: 04.46$ & 1.013 & $\mathrm{O}$ II \\
\hline obj025 & 21:52:00.381 & $+06: 25: 20.46$ & 1.052 & $\mathrm{O}$ II \\
\hline obj026 & 21:51:59.549 & $+06: 25: 39.06$ & 1.053 & $\mathrm{O}$ II \\
\hline obj027 & 21:52:02.380 & $+06: 24: 58.06$ & 0.185 & $\mathrm{O}$ III, $\mathrm{H}_{\beta}, \mathrm{H}_{\alpha}, \mathrm{N}$ II \\
\hline obj028 & $21: 51: 58.583$ & $+06: 25: 34.26$ & $\cdots$ & 8413.87 \\
\hline obj029 & 21:52:00.904 & $+06: 24: 50.26$ & 1.302 & $\mathrm{O}$ II \\
\hline obj030 & 21:52:00.019 & $+06: 25: 13.26$ & 1.318 & $\mathrm{O}$ II \\
\hline obj031 & 21:51:59.952 & $+06: 25: 15.46$ & 1.318 & $\mathrm{O}$ II \\
\hline obj032 & $21: 51: 58.355$ & $+06: 25: 03.06$ & 1.349 & $\mathrm{O}$ II \\
\hline obj033 & 21:51:58.489 & $+06: 24: 59.06$ & $\cdots$ & 8757.32 \\
\hline obj034 & $21: 52: 02.354$ & $+06: 25: 15.46$ & 1.362 & $\mathrm{O}$ II \\
\hline obj035 & $21: 51: 58.355$ & $+06: 25: 23.66$ & 1.403 & $\mathrm{O}$ II \\
\hline obj036 & 21:52:00.435 & $+06: 25: 13.46$ & 1.430 & $\mathrm{O}$ II \\
\hline obj037 & 21:52:00.623 & $+06: 25: 15.86$ & 1.430 & $\mathrm{O}$ II \\
\hline obj038 & 21:52:01.629 & $+06: 25: 24.06$ & 1.431 & $\mathrm{O}$ II \\
\hline obj039 & 21:52:00.972 & $+06: 25: 33.06$ & 1.433 & $\mathrm{O}$ II \\
\hline obj040 & 21:52:00.703 & $+06: 25: 43.06$ & 1.435 & $\mathrm{O}$ II \\
\hline obj041 & 21:52:00.180 & $+06: 25: 41.26$ & 1.432 & $\mathrm{O}$ II \\
\hline
\end{tabular}

Note. Within these 41 galaxies, 40 have identified emission lines.

system geometry. To constrain the ejected mass rate probed by the quasar LOS, we use Equation (5) from Bouché et al. (2012) and Schroetter et al. (2015):

$$
\begin{aligned}
& \dot{M}_{\mathrm{out}} \approx \mu \cdot N_{\mathrm{H}}(b) \cdot b \cdot V_{\mathrm{out}} \cdot \frac{\pi}{2} \cdot \theta_{\max } \\
& \frac{\dot{M}_{\mathrm{out}}}{0.5 M_{\odot} \mathrm{yr}^{-1}} \approx \frac{\mu}{1.5} \cdot \frac{N_{\mathrm{H}}(b)}{10^{19} \mathrm{~cm}^{-2}} \\
& \cdot \frac{b}{25 \mathrm{kpc}} \cdot \frac{V_{\mathrm{out}}}{200 \mathrm{~km} \mathrm{~s}^{-1}} \cdot \frac{\theta_{\max }}{30^{\circ}}
\end{aligned}
$$

$\mu$ being the mean atomic weight, $b$ the impact parameter, $\theta_{\max }$ the cone opening angle, ${ }^{21} V_{\text {out }}$ the outflow velocity and $N_{\mathrm{H}}(b)$ is the gas column density at the $b$ distance.

$\overline{21}$ We remind the reader that $\theta_{\max }$ is defined in Section 4.

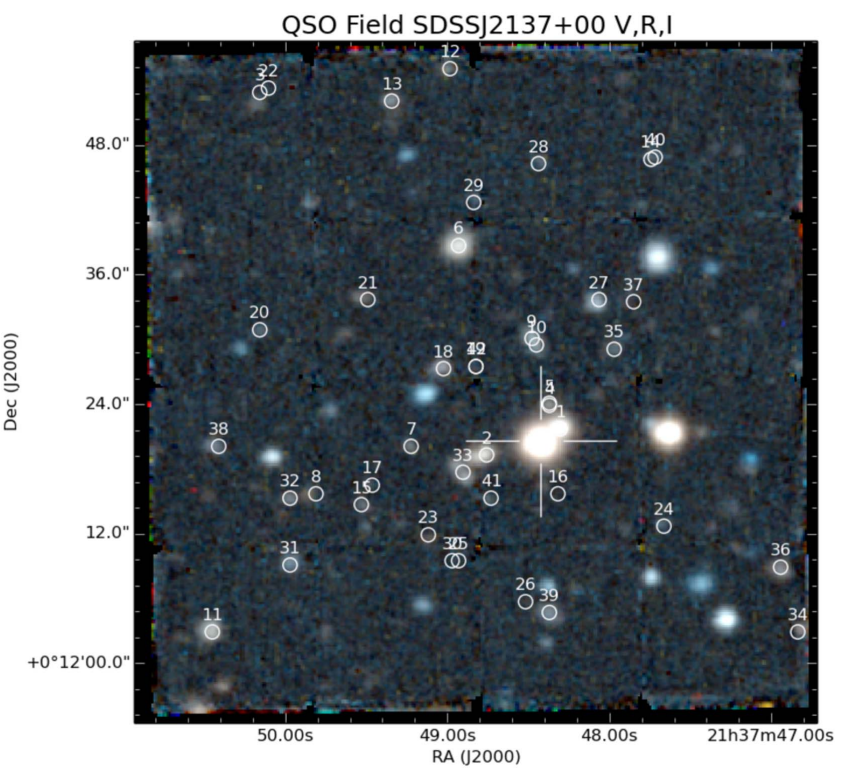

Figure 14. Red giant branch image of the J213748+0012 field with identifications of emission-detected galaxies. The white cross points out the quasar location. Circles represent emission-detected galaxies corresponding to Table 9. Not all the galaxy-like spots are circled on the image. These spots are either stars or galaxies with a continuum, but without obvious emission line.

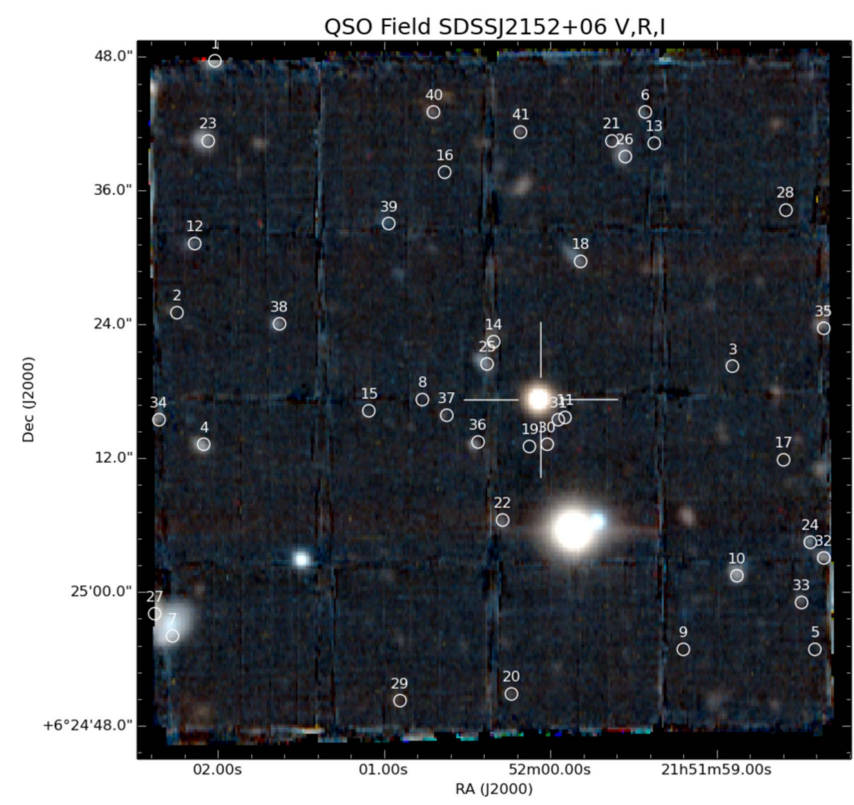

Figure 15. Same as Figure 14, but for the J215200+0625 quasar field. Again, the white cross shows the quasar location, and galaxies with emission lines are circled and listed in Table 10.

The only parameter, which is yet to be constrained, is the gas column density $N_{\mathrm{H}}(b)$. To do that, we use the empirical relation between the neutral gas column density and the $\mathrm{Mg}$ II $\lambda 2796$ $\mathrm{REW} W_{r}^{\lambda 2796}$ (Equation 6 of Ménard \& Chelouche 2009):

$$
\begin{aligned}
\log \left(N_{\mathrm{HI}}\right)\left(\mathrm{cm}^{-2}\right)= & \log [(3.06 \pm 0.55) \\
& \left.\times 10^{19} \times\left(W_{r}^{\lambda 2796}\right)^{1.7 \pm 0.26}\right] .
\end{aligned}
$$

To compute the errors, we assume a Gaussian error distribution. As described in Schroetter et al. (2015), for regions with $\mathrm{H}$ I column density above $\log \left(N_{\mathrm{H}}\right)=19.5$, the ionized gas contribution is negligible. Also argued by Jenkins 
(2009), if this column density is above this limit, one can use the correlation between the $\mathrm{Mg}$ II equivalent width and $N_{\mathrm{H} \text { I }}$ as a proxy for the $N_{\mathrm{H}}$ gas column density. For the wind-pair $\mathrm{J} 215200+0625 \mathrm{G} 2$, we have a gas column density of $\log \left(N_{\mathrm{H}}\right) \approx 19.7 \pm 0.07$.

Another aspect of outflow properties is whether the outflowing gas is able to escape from the galaxy gravitational well. To determine this, we derive the escape velocity $V_{\text {esc }}$ for the J215200+0625G2 galaxy. The escape velocity for an isothermal sphere is defined by Equation (7) (Veilleux et al. 2005).

$$
V_{\mathrm{esc}}=V_{\max } \cdot \sqrt{2\left[1+\ln \left(\frac{R_{\mathrm{vir}}}{r}\right)\right]}
$$

$V_{\max }$ being the maximum rotation velocity of the galaxy and $R_{\mathrm{vir}}$ its virial radius. The virial radius of the galaxies can be defined as $R_{\text {vir }} \approx V_{\max } / 10 H(z)$, where $H(z)$ is the Hubble parameter at redshift $z$. In Table 8, we compare the outflow velocity with the escape velocity for the wind-pair. This ratio $V_{\text {out }} / V_{\text {esc }}$ of 0.52 shows that the outflowing material is not able to reach the escape velocity and thus will be likely to fall back onto the galaxy, assuming we are tracing the gas going out of the galaxy. One can ask whether we are already tracing the gas falling back onto the galaxy. If this is the case, we should see another opposite component (with respect to the systemic velocity) in the absorption profile corresponding to the outflowing gas.

Table 8 also lists the estimated outflow rate. The errors on the ejected mass rate $\dot{M}_{\text {out }}$ are dominated by the ones on the gas column density $N_{\mathrm{H} \text { I }}$ and the SFR.

From the outflow rate, we compute the mass loading factor $\eta$ by comparing it to the SFR $\left(\eta=\dot{M}_{\text {out }} /\right.$ SFR $)$. For our SDSS $\mathbf{J} 215200+0625 G 2$ pair, we used the empty cone model to reproduce the absorption profile with an inner cone opening angle $\theta_{\text {in }}$ of $7^{\circ}$. To be consistent with the other cases, we give two solutions for this galaxy-quasar pair: one with the filled cone and one with the inner cone subtracted.

Figure 13 shows the loading factor $\eta$ as a function of halo mass and maximum rotational velocity $V_{\max }$ for this work and previous similar studies (Bouché et al. 2012; Kacprzak et al. 2014; Schroetter et al. 2015). The derived loading factor for galaxy SDSS J215200+0625G2 follows the same trend as the others. The red arrow shows the loading factor for the subtracted mass from the low-density inner cone.

MUSE allows us to probe galaxies with an impact parameter larger than before with an IFU. But, in Figure 13, we caution the reader that the loading factor for galaxies with impact parameters larger than $60 \mathrm{kpc}$ are less reliable, because of the time needed for the gas to travel from the galaxy to the quasar LOS ( 400 Myr at $V_{\text {out }} \approx 150 \mathrm{~km} \mathrm{~s}^{-1}$ with $b=60 \mathrm{kpc}$ ). A major limitation for the comparison between data and models in Figure 13, is that $\eta$ in simulations are usually measured on a scale of a few kpc away from the galaxy, which is one order of magnitude lower than most of the observations (tens of $\mathrm{kpc}$ ).

\section{SUMMARY}

We present results on two GTO VLT/MUSE fields in which we searched for galaxy-quasar pairs. These fields were selected from the SDSS database, where we searched for multiple $\mathrm{Mg}$ II absorbers, with $z \approx 0.8-1.4$ and $W_{r}^{\lambda 2796}>0.5 \AA$, in the quasar spectra. Out of eight $\mathrm{Mg}$ II absorptions in the quasar spectra of these two fields, we detect six redshift-corresponding SFGs. For these two fields (J213748+1112 and J215200+0625), we also have high-resolution spectra of the quasars from the VLT/ UVES instrument. In each of these two fields, we detected more than 40 emitters in the $1^{\prime} \times 1^{\prime}$ MUSE field of view (see the Appendix). We focused on galaxies at $\mathrm{Mg}$ II absorption redshifts in the quasar spectra and for which the associated quasar LOS is aligned with their minor axis $\left(\alpha>55^{\circ}\right)$ and is thus likely to probe outflowing materials (wind-pairs). Among the six detected SFGs, one is likely to be a wind-pair due to its orientation with respect to its relative quasar.

In summary, thanks to our new GTO VLT/MUSE and VLT/UVES data, MUSE allows us to detect galaxies far away from their associated quasar $(\sim 100 \mathrm{kpc})$ compared to previous similar works (i.e., Bouché et al. 2012; Kacprzak et al. 2014; Schroetter et al. 2015). For the wind-pair SDSS J215200 $+0625 \mathrm{G} 2$, we found that the outflow velocity $V_{\text {out }}$ is $\approx 150 \mathrm{~km} \mathrm{~s}^{-1}$. The outflowing gas is likely to stay inside the gravitational well of the galaxy and the loading factor is $\eta \approx 0.7$. We showed a gap in velocities in the absorption profile, which led to a low-density inner cone modeling. At this point, we have outflowing constraints for one galaxy, but we showed that MUSE is able to provide very good data and will play a fundamental role in this field.

MUSE allowed us to probe multiple galactic wind cases at the same time and enhance the number of cases with only two quasar fields. We also have a case of low-density inner cone, which opens discussions on geometrical properties of outflowing materials. The MEGAFLOW sample is currently growing and is successful in detecting galaxies in each quasar field ( $\approx 84 \%$ detection). Future work will be done with a lot more observation with MUSE+UVES, and in a short time, the MEGAFLOW sample should be large enough to produce statistical results on outflow properties.

N.B. acknowledges support from a Career Integration Grant (CIG) (PCIG11-GA-2012-321702) within the 7th European Community Framework Program. This work has been carried out thanks to the support of the ANR FOGHAR (ANR-13BS05-0010-02), the OCEVU Labex (ANR-11-LABX-0060) and the $A^{*}$ MIDEX project (ANR-11-IDEX-0001-02) funded by the "Investissements d'Avenir" French government program managed by the ANR. This work received financial support from the European Research Council under the European Unions Seventh Framework Programme (FP7/2007-2013)/ ERC Grant agreement 278594-GasAroundGalaxies.

\section{APPENDIX \\ MUSE FIELDS' EMITTERS DETECTION}

For completeness, we looked for these emitters by visual inspection and found 42 galaxies with emission lines in each of these two fields (see Table 9 for SDSS J213748+0012 and Table 10 for SDSS J215200+0625).

\section{REFERENCES}

Aguirre, A., Hernquist, L., Schaye, J., et al. 2001, ApJ, 561, 521 Alam, S., Albareti, F. D., Allende Prieto, C., et al. 2015, ApJS, 219, 12 Bacon, R., Bauer, S., Boehm, P., et al. 2006, Proc. SPIE, 6269, 62690J Bacon, R., Bauer, S., Brau-Nogué, S., et al. 2009, ASSP, 9, 331 Barai, P., Monaco, P., Murante, G., Ragagnin, A., \& Viel, M. 2015, MNRAS, 447, 266

Behroozi, P. S., Wechsler, R. H., \& Conroy, C. 2013, ApJ, 770, 57 
Booth, C. M., Agertz, O., Kravtsov, A. V., \& Gnedin, N. Y. 2013, ApJL, 777, L16

Bordoloi, R., Lilly, S. J., Kacprzak, G. G., \& Churchill, C. W. 2014, ApJ, 784, 108

Bordoloi, R., Lilly, S. J., Knobel, C., et al. 2011, ApJ, 743, 10

Bouché, N., Carfantan, H., Schroetter, I., Michel-Dansac, L., \& Contini, T. 2015a, GalPaK 3D: Galaxy Parameters and Kinematics Extraction from 3D Data, Astrophysics Source Code Library, ascl:1501.014

Bouché, N., Carfantan, H., Schroetter, I., Michel-Dansac, L., \& Contini, T. 2015b, AJ, 150, 92

Bouché, N., Finley, H., Schroetter, I., et al. 2016, ApJ, 820, 121

Bouché, N., Hohensee, W., Vargas, R., et al. 2012, MNRAS, 426, 801

Chabrier, G. 2003, PASP, 115, 763

Charlot, S., Kauffmann, G., Longhetti, M., et al. 2002, MNRAS, 330, 876

Chen, H.-W., Wild, V., Tinker, J. L., et al. 2010, ApJL, 724, L176

Chisholm, J., Tremonti, C. A., Leitherer, C., et al. 2015, ApJ, 811, 149

Chisholm, J., Tremonti, C. A., Leitherer, C., Chen, Y., \& Wofford, A. 2016, arXiv: 1601.05090

Dekel, A., \& Silk, J. 1986, ApJ, 303, 39

Dekker, H., D’Odorico, S., Kaufer, A., Delabre, B., \& Kotzlowski, H. 2000, Proc. SPIE, 4008, 534

Epinat, B., Tasca, L., Amram, P., et al. 2012, A\&A, 539, A92

Fox, A. J., Bordoloi, R., Savage, B. D., et al. 2015, ApJL, 799, L7

Guo, Q., White, S., Li, C., \& Boylan-Kolchin, M. 2010, MNRAS, 404, 1111

Heckman, T. M., Alexandroff, R. M., Borthakur, S., Overzier, R., \& Leitherer, C. 2015, ApJ, 809, 147

Heckman, T. M., Armus, L., \& Miley, G. K. 1990, ApJS, 74, 833

Heckman, T. M., Lehnert, M. D., Strickland, D. K., \& Armus, L. 2000, ApJS, 129,493

Hopkins, P. F. 2015, MNRAS, 450, 53

Hopkins, P. F., Kereš, D., Oñorbe, J., et al. 2014, MNRAS, 445, 581

Jenkins, E. B. 2009, ApJ, 700, 1299

Kacprzak, G. G., Churchill, C. W., Barton, E. J., \& Cooke, J. 2011a, ApJ, 733, 105

Kacprzak, G. G., Churchill, C. W., Evans, J. L., Murphy, M. T., \& Steidel, C. C. 2011b, MNRAS, 416, 3118

Kacprzak, G. G., Churchill, C. W., \& Nielsen, N. M. 2012, ApJL, 760, L7

Kacprzak, G. G., Martin, C. L., Bouché, N., et al. 2014, ApJL, 792, L12

Kennicutt, R. C., Jr. 1998, ApJ, 498, 541

Kewley, L. J., Geller, M. J., \& Jansen, R. A. 2004, AJ, 127, 2002

Lan, T.-W., Ménard, B., \& Zhu, G. 2014, ApJ, 795, 31

Lanzetta, K. M., \& Bowen, D. 1990, ApJ, 357, 321

Lehnert, M. D., \& Heckman, T. M. 1996, ApJ, 472, 546

Martin, C. L. 1998, ApJ, 506, 222

Martin, C. L. 1999, ApJ, 513, 156

Martin, C. L. 2005, ApJ, 621, 227

Martin, C. L., Kobulnicky, H. A., \& Heckman, T. M. 2002, ApJ, 574, 663
Martin, C. L., Shapley, A. E., Coil, A. L., et al. 2012, ApJ, 760, 127

Martin, C. L., Shapley, A. E., Coil, A. L., et al. 2013, ApJ, 770, 41

Ménard, B., \& Chelouche, D. 2009, MNRAS, 393, 808

Mo, H. J., \& White, S. D. M. 2002, MNRAS, 336, 112

Moster, B. P., Naab, T., \& White, S. D. M. 2013, MNRAS, 428, 3121

Moster, B. P., Somerville, R. S., Maulbetsch, C., et al. 2010, ApJ, 710, 903

Muratov, A. L., Keres, D., Faucher-Giguere, C.-A., et al. 2015, MNRAS, 454, 2691

Murray, N., Quataert, E., \& Thompson, T. A. 2005, ApJ, 618, 569

Muzahid, S., Kacprzak, G. G., Churchill, C. W., et al. 2015, ApJ, 811, 132

Nestor, D. B., Turnshek, D. A., \& Rao, S. M. 2005, ApJ, 628, 637

Nielsen, N. M., Churchill, C. W., \& Kacprzak, G. G. 2013, ApJ, 776, 115

Oppenheimer, B. D., \& Davé, R. 2006, MNRAS, 373, 1265

Oppenheimer, B. D., Davé, R., Kereš, D., et al. 2010, MNRAS, 406, 2325

Papastergis, E., Cattaneo, A., Huang, S., Giovanelli, R., \& Haynes, M. P. 2012, ApJ, 759, 138

Pettini, M., Rix, S. A., Steidel, C. C., et al. 2002, ApJ, 569, 742

Ross, N. P., Myers, A. D., Sheldon, E. S., et al. 2012, ApJS, 199, 3

Rubin, K. H. R., Prochaska, J. X., Koo, D. C., et al. 2014, ApJ, 794, 156

Rubin, K. H. R., Weiner, B. J., Koo, D. C., et al. 2010, ApJ, 719, 1503

Rupke, D. S., Veilleux, S., \& Sanders, D. B. 2005, ApJS, 160, 115

Salem, M., \& Bryan, G. L. 2014, MNRAS, 437, 3312

Salpeter, E. E. 1955, ApJ, 121, 161

Schaye, J., Crain, R. A., Bower, R. G., et al. 2015, MNRAS, 446, 521

Schaye, J., Dalla Vecchia, C., Booth, C. M., et al. 2010, MNRAS, 402, 1536

Schroetter, I., Bouché, N., Péroux, C., et al. 2015, ApJ, 804, 83

Soto, K. T., Lilly, S. J., Bacon, R., Richard, J., \& Conseil, S. 2016a, MNRAS, 458,3210

Soto, K. T., Lilly, S. J., Bacon, R., Richard, J., \& Conseil, S. 2016b, ZAP: Zurich Atmosphere Purge, Astrophysics Source Code Library, ascl: 1602.003

Springel, V., \& Hernquist, L. 2003, MNRAS, 339, 289

Springel, V., White, S. D. M., Jenkins, A., et al. 2005, Natur, 435, 629

Steidel, C. C. 1995, in QSO Absorption Lines, ed. G. Meylan (Berlin: Springer), 139

Torrey, P., Vogelsberger, M., Genel, S., et al. 2014, MNRAS, 438, 1985

Veilleux, S., Cecil, G., \& Bland-Hawthorn, J. 2005, ARA\&A, 43, 769

Veilleux, S., \& Rupke, D. 2002, AAONw, 99, 6

Vogelsberger, M., Genel, S., Springel, V., et al. 2014, Natur, 509, 177

Weilbacher, P. M., Streicher, O., Urrutia, T., et al. 2014, in ASP Conf. Ser. 485, Astronomical Data Analysis Software and Systems XXIII, ed. N. Manset \& P. Forshay (San Francisco, CA: ASP), 451

Werk, J. K., Prochaska, J. X., Thom, C., et al. 2013, ApJS, 204, 17

Wood, C. M., Tremonti, C. A., Calzetti, D., et al. 2015, MNRAS, 452,2712

Zahid, H. J., Torrey, P., Vogelsberger, M., et al. 2014, Ap\&SS, 349, 873

Zhu, G., \& Ménard, B. 2013, ApJ, 770, 130 